\title{
ANALYSIS OF WHITE NOISE LIMITS FOR STOCHASTIC SYSTEMS WITH TWO FAST RELAXATION TIMES*
}

\author{
G. A. PAVLIOTIS ${ }^{\dagger}$ AND A. M. STUART ${ }^{\ddagger}$
}

\begin{abstract}
In this paper we present a rigorous asymptotic analysis for stochastic systems with two fast relaxation times. The mathematical model analyzed in this paper consists of a Langevin equation for the particle motion with time-dependent force constructed through an infinite dimensional Gaussian noise process. We study the limit as the particle relaxation time as well as the correlation time of the noise tend to zero, and we obtain the limiting equations under appropriate assumptions on the Gaussian noise. We show that the limiting equation depends on the relative magnitude of the two fast time scales of the system. In particular, we prove that in the case where the two relaxation times converge to zero at the same rate there is a drift correction, in addition to the limiting Itô integral, which is not of Stratonovich type. If, on the other hand, the colored noise is smooth on the scale of particle relaxation, then the drift correction is the standard Stratonovich correction. If the noise is rough on this scale, then there is no drift correction. Strong (i.e., pathwise) techniques are used for the proof of the convergence theorems.
\end{abstract}

Key words. white noise limits, Ornstein-Uhlenbeck process, Kraichnan model, Wong-Zakai theorem

AMS subject classifications. 60H10, 60H15, 60H30, 60G15

DOI. $10.1137 / 040610507$

1. Introduction. Many physical systems are subject to either additive or multiplicative noise. The dynamics of such systems are quite often adequately described by systems of SDEs. There are various applications where the noise in the physical system under investigation has a nontrivial spatio-temporal structure and where it is not realistic to model it as a white noise process. The term colored noise is used for such a noise process.

It is a well-known result that if we approximate white noise by a smooth, colored process, then at the limit, as the correlation time of the approximation tends to zero, the smoothed stochastic integral converges to the Stratonovich stochastic integral $[4,32],[2$, Ch. 10]. To be precise, consider the SDE (written here in one dimension for simplicity)

$$
\dot{x}=b(x)+\frac{f(x) \eta\left(t / \epsilon^{2}\right)}{\epsilon},
$$

where $b(x), f(x)$ are Lipschitz continuous and $\eta(t)$ is a continuous mean zero Gaussian process with $\mathbb{E}(\eta(t) \eta(s))=\frac{1}{2} e^{-|t-s|}$. Then the results of $[4,32]$ imply that, as $\epsilon$

*Received by the editors June 24, 2004; accepted for publication (in revised form) November 23, 2004; published electronically June 3, 2005. This work was supported by the Engineering and Physical Sciences Research Council.

http://www.siam.org/journals/mms/4-1/61050.html

${ }^{\dagger}$ Mathematics Institute, Warwick University, Coventry CV4 7AL, United Kingdom. Current address: Department of Mathematics, Imperial College London, London SW7 2AZ, United Kingdom (g.pavliotis@imperial.ac.uk).

${ }^{\ddagger}$ Mathematics Institute, Warwick University, Coventry CV4 7AL, United Kingdom (stuart@ maths.warwick.ac.uk). 
converges to 0 , the solution of (1.1) converges weakly to $X(t)$, which satisfies ${ }^{1}$

$$
\dot{X}(t)=b(X)+\frac{1}{2} f(X) f^{\prime}(X)+f(X) \dot{\beta} .
$$

Here $\beta(t)$ denotes a standard one-dimensional Brownian motion. The term $\frac{1}{2} f f^{\prime}$ is sometimes referred to as the Stratonovich correction. This result has been extended in various ways, including the case of multiple Itô integrals [19], as well as linear $[5,6,10,20]$ and semilinear SPDEs; see [33] and the references therein. Moreover, the case of infinite dimensional noise has also been studied $[6,8,10]$. In the context of the theory of turbulent diffusion, the results of the aforementioned papers are concerned with the convergence of rapidly decorrelating in time velocity fields to the Kraichnan model for passive tracers [16].

The main interest of this work is to extend these results to situations where inertial effects are taken into account. For motivation, consider the motion of a particle with relaxation time $\tau$ under the influence of a force field $b(x)$ and subject to dissipation and colored multiplicative noise:

$$
\tau \ddot{x}=b(x)-\dot{x}+\frac{f(x) \eta\left(t / \epsilon^{2}\right)}{\epsilon} .
$$

We are interested in analyzing the limit of (1.3) as both $\tau$ and $\epsilon$ tend to 0 . It should be expected that these two limits do not commute. To see this, first let $\epsilon \rightarrow 0$ while keeping $\tau$ fixed to obtain the $\mathrm{SDE}^{2}$

$$
\tau \ddot{x}=b(x)-\dot{x}+f(x) \dot{\beta} .
$$

Now taking the limit as $\tau \rightarrow 0$ leads to the Itô SDE [22, Ch. 10]

$$
\dot{x}=b(x)+f(x) \dot{\beta} .
$$

On the other hand, if we first take the limit as $\tau \rightarrow 0$ and then let $\epsilon \rightarrow 0$, we end up with (1.2).

Because of this lack of commutativity, it is not clear what the limiting equation should be as we let $\epsilon$ and $\tau$ both tend to 0 at the same time. This is sometimes referred to as the Itô versus Stratonovich problem in the physics literature [26]. The correct form of the limiting SDE and, in particular, the presence or otherwise of a drift correction term in addition to the limiting Itô integral - the noise induced drift - is of particular importance in the theory of Brownian motors [26], noise induced phase transitions [11, 15, 21], and the dynamics of fronts [28].

The purpose of this paper is to investigate the Itô versus Stratonovich problem rigorously. Let us now discuss the main results of this paper in the one-dimensional setting. The one-dimensional version of the model considered in this paper reads

$$
\begin{gathered}
\tau_{0} \epsilon^{\gamma} \ddot{x}=b(x)-\dot{x}+\frac{f(x) \eta\left(t / \epsilon^{2}\right)}{\epsilon}, \\
\dot{\eta}=-\alpha \eta+\sqrt{\lambda} \dot{\beta},
\end{gathered}
$$

\footnotetext{
${ }^{1}$ Throughout the paper we will use the notation $\int_{0}^{t} f(x(s)) d \beta(s)$ (respectively, $\left.f(x(t)) \dot{\beta}(t)\right)$ to denote the Itô stochastic integral (respectively, differential) and $\int_{0}^{t} f(x(s)) \circ d \beta(s)$ (respectively, $f(x(t)) \circ \dot{\beta}(t))$ for the Stratonovich stochastic integral (respectively, differential). Furthermore, we will refer to an Itô or Stratonovich SDE depending on how we choose to interpret the stochastic integral in the equation.

${ }^{2}$ It is easy to check that in this case there is no Stratonovich correction to the Itô integral, because of the regularity of $x$.
} 
where $\alpha, \lambda$, and $\tau_{0}$ are positive $\mathcal{O}(1)$ parameters and $\gamma \in(0, \infty)$. In this paper we show that three possible limiting equations result, depending on the magnitude of the particle relaxation time relative to that of the noise correlation time, i.e., depending on the exponent $\gamma$. In particular, for $\gamma \in(0,2)$ we show that the limiting equation is the Itô SDE

$$
\dot{X}(t)=b(X)+\frac{\sqrt{\lambda}}{\alpha} f(X) \dot{\beta} .
$$

For $\gamma \in(2, \infty)$ we obtain, at the limit $\epsilon \rightarrow 0$, the Stratonovich SDE

$$
\begin{aligned}
\dot{X}(t) & =b(X)+\frac{\sqrt{\lambda}}{\alpha} f(X) \circ \dot{\beta} \\
& =b(X)+\frac{\lambda}{2 \alpha^{2}} f(X) f^{\prime}(X)+\frac{\sqrt{\lambda}}{\alpha} f(X) \dot{\beta} .
\end{aligned}
$$

For $\gamma=2$ the limiting SDE can be interpreted in neither the Itô nor the Stratonovich sense; we obtain

$$
\dot{X}(t)=b(X)+\frac{\lambda}{2 \alpha^{2}\left(1+\tau_{0} \alpha\right)} f(X) f^{\prime}(X)+\frac{\sqrt{\lambda}}{\alpha} f(X) \dot{\beta} .
$$

Let us make some remarks concerning (1.7). If we define the stochastic integral

$$
\int_{0}^{t} f(X(s)) \widehat{o} d \beta(s):=\frac{\lambda}{2 \alpha^{2}\left(1+\tau_{0} \alpha\right)} \int_{0}^{t} f(X(s)) f^{\prime}(X(s)) d s+\frac{\sqrt{\lambda}}{\alpha} \int_{0}^{t} f(X(s)) d \beta(s),
$$

then this integral obeys neither the Itô nor the Stratonovich (i.e., Newton-Leibnitz) calculus. Let us now define the stochastic integral in (1.8) as the limit of Riemann sums

$$
\int_{0}^{t} f(X(s)) \widehat{o} d \beta(s) \approx \frac{\sqrt{\lambda}}{\alpha} \sum_{j=1}^{N}\left(\mu f\left(X\left(s_{j}\right)\right)+(1-\mu) f\left(X\left(s_{j-1}\right)\right)\left(\beta\left(s_{j}\right)-\beta\left(s_{j-1}\right)\right),\right.
$$

with $\mu \in[0,1]$. The stochastic integral (1.8) corresponds to the choice $\mu=\frac{1}{2\left(1+\tau_{0} \alpha\right)}$. As is well known (see, e.g., [23]), the Itô integral corresponds to the choice $\mu=0$, whereas the Stratonovich integral corresponds to $\mu=\frac{1}{2}$. Notice that letting $\tau_{0}$ in (1.7) vary in $(0, \infty)$ interpolates between these two well-known integrals.

The one-dimensional model (1.4) was studied by Graham and Schenzle in [13] using formal singular perturbation analysis for the corresponding Fokker-Planck equation in the spirit of [4]. Similar questions to the one studied in this paper were investigated by Givon and Kupferman in [12] for SDE limits of discrete dynamical systems with scale separation. Specific examples were presented where the limiting SDE is neither of Itô nor of Stratonovich type. A formal derivation of the results reported in this paper, together with extensive numerical simulations, were presented in [18].

In this paper we base our rigorous derivation of the limiting SDE for the infinite dimensional version of (1.4) - see (2.4) below — using the pathwise techniques developed in [24], following the work of [8]. Our method enables us to treat the infinite dimensionality of the noise in a rather straightforward way and, in addition, to prove 
strong convergence results. Furthermore, we are able to prove upper bounds on the convergence rate in $L^{p}(\Omega ; C([0, T] ; \mathbb{R}))$. The numerical results reported in [18] indicate that the upper bounds are in fact sharp.

This paper is organized as follows. In section 2 we describe the equations that we will be studying and present the convergence theorems. In section 3 we present various preliminary results which are necessary for the proof of our convergence theorems. In section 4 we show that the structure of the limiting equations depends crucially on $\gamma$. Our convergence theorems are proved in section 5. In section 6 we present two applications of the convergence theorems, with particular emphasis on the inertial particles problem considered in $[24,29,30]$. Finally, section 7 is devoted to some concluding remarks.

2. Description of the model and statement of main results. In some of the applications of interest to us the driving colored noise is infinite dimensional. This arises, for instance, in Gaussian random field models of turbulence such as those pioneered by Kraichnan [16] and in generalizations to include noise correlation times $[6,7,10,17,29,30]$. Such applications are described in section 6 . In this section we formulate the problem for infinite dimensional driving noises and state our main results.

2.1. The model. We consider the Langevin dynamics for a particle moving in $\mathbb{R}^{d}, d \geq 1$, under the influence of a forcing term $b(x)$ and a rapidly decorrelating in time random field $v(x, t)$ :

$$
\epsilon^{\gamma} \tau_{0} \ddot{x}=b(x)+\frac{v\left(x, t / \epsilon^{2}\right)}{\epsilon}-\dot{x}, \quad x \in \mathbb{R}^{d},
$$

where $\gamma \in(0, \infty)$ and $\epsilon \ll 1$. The field $v(x, t)$ is a generalized Ornstein-Uhlenbeck $(\mathrm{OU})$ process. This is a mean zero, Gaussian process which can be constructed as the solution of the vector valued SPDE

$$
d v=-\widehat{A} v d t+d \widehat{W}
$$

Here we take $\widehat{A}: D(\widehat{A}) \rightarrow\left(L^{2}(\Omega)\right)^{d}$, where $\Omega \subset \mathbb{R}^{d}$ and $\widehat{W}$ is a $\widehat{Q}$-Wiener process on $H=\left(L^{2}(\Omega)\right)^{d}$. We assume that $\widehat{A}$ is a strictly positive self-adjoint operator on the Hilbert space $H$ and that, furthermore, it has the same eigenfunctions $\left\{f_{k}\right\}_{k=1}^{\infty}$ as $\widehat{Q}$ :

$$
\widehat{A} f_{k}=\alpha_{k} f_{k}, \quad \widehat{Q} f_{k}=\lambda_{k} f_{k} .
$$

We now assume that there exist vectors $h_{k} \in \mathbb{R}^{d}$ and positive definite self-adjoint operators $A, Q$ on $L^{2}(\Omega)$ such that

$$
f_{k}=h_{k} \phi_{k}, \quad A \phi_{k}=\alpha_{k} \phi_{k}, \quad Q \phi_{k}=\lambda_{k} \phi_{k} .
$$

Using this we can write

$$
v(x, t)=f(x) \eta(t)=\sum_{k=1}^{\infty} h_{k} \phi_{k}(x) \eta_{k}(t),
$$

where $\eta(t): \ell_{2} \rightarrow \mathbb{R}$ is defined through the equation

$$
d \eta=-A \eta d t+d W
$$


Here, abusing notation, we have used $A, Q \in L\left(\ell_{2}\right)$ with

$$
A=\operatorname{diag}\left\{\alpha_{k}\right\}, \quad Q=\operatorname{diag}\left\{\lambda_{k}\right\} .
$$

Furthermore, $W$ is a $Q$-Wiener process on $\ell^{2}$ :

$$
W(t)=\sum_{k=1}^{\infty} \sqrt{\lambda_{k}} e_{k} \beta_{k}(t)
$$

with $\left\{e_{k}\right\}_{k=1}^{\infty}$ being the standard basis in $\ell_{2}$ and $\beta_{k}(t)$ the mutually independent standard one-dimensional Brownian motions. We remark that, for each fixed $x, f$ is a linear operator from $\ell_{2}$ to $\mathbb{R}^{d}: f \in L\left(\ell^{2}, \mathbb{R}^{d}\right)$.

Now using the fact that $\beta(c t)=\sqrt{c} \beta(t)$ in law, we can finally write our model in the following form:

$$
\begin{gathered}
\epsilon^{\gamma} \ddot{x}=b(x)+\frac{v(x, t)}{\epsilon}-\dot{x}, \\
v(x, t)=f(x) \eta(t), \\
d \eta=-\frac{1}{\epsilon^{2}} A \eta d t+\frac{1}{\epsilon} d W .
\end{gathered}
$$

To simplify the notation we have set $\tau_{0}=1$ in (2.4a). In what follows we will use both notations $v(x, t)$ and $f(x) \eta(t)$ for the random field.

2.2. Statement of main results. Now our goal is to obtain the limiting equations of motion as $\epsilon \rightarrow 0$. In order to prove our convergence theorems we will need to impose various conditions on the spectrum of the Wiener process, the eigenvalues of the operator $A$, the eigenfunctions $\left\{\phi_{k}(x)\right\}_{k=1}^{\infty}$, and the drift term $b(x)$. The conditions that we have to impose are more severe for $\gamma \geq 2$, since in this parameter regime we will need more integrations by parts in order to obtain the limiting equations.

We will use the notation $\|\cdot\|$ to denote the Euclidean norm in $\mathbb{R}^{d}$. Subscripts with commas will be used to denote partial differentiation.

As has already been mentioned, we take $A$ to be a self-adjoint, positive operator on $L^{2}(\Omega)$. We assume that the eigenvalues $\left\{\alpha_{k}\right\}_{k=1}^{\infty}$ of $A$ satisfy

$$
\cdots \geq \alpha_{k+1} \geq \alpha_{k} \geq \omega>0, \quad \sum_{k=1}^{\infty} \frac{\lambda_{k}}{2 \alpha_{k}}<\infty .
$$

The eigenfunctions of $A$ are normalized so that their $L^{2}(\Omega)$ norm is set to 1 : $\left\|\phi_{k}\right\|_{L^{2}(\Omega)}$ $=1$. Moreover, for $\gamma \in(0,2)$ we assume that there exist constants $C>0, \alpha, \beta$ such that

$$
\left\{\begin{array}{r}
\phi_{k}(x) \in C_{b}^{2}(\Omega), \quad k=1,2, \ldots, \\
\left\|\phi_{k}(x)\right\|_{L^{\infty}(\Omega)} \leq C \alpha_{k}^{\alpha},\left\|D \phi_{k}(x)\right\|_{L^{\infty}(\Omega)} \leq C \alpha_{k}^{\beta} .
\end{array}\right.
$$

The conditions for $\gamma \geq 2$ are more severe. We assume that there exist constants $C>0, \alpha, \beta, \gamma, \delta$ such that

$$
\left\{\begin{array}{r}
\phi_{k}(x) \in C_{b}^{3}(\Omega), \quad k=1,2, \ldots, \\
\left\|\phi_{k}(x)\right\|_{L^{\infty}(\Omega)} \leq C \alpha_{k}^{\alpha},\left\|D \phi_{k}(x)\right\|_{L^{\infty}(\Omega)} \leq C \alpha_{k}^{\beta}, \\
\left\|D^{2} \phi_{k}(x)\right\|_{L^{\infty}(\Omega)} \leq C \alpha_{k}^{\gamma},\left\|D^{3} \phi_{k}(x)\right\|_{L^{\infty}(\Omega)} \leq C \alpha_{k}^{\delta} .
\end{array}\right.
$$


REMARK 2.1. At this level of generality and, in particular, since we do not make any specific assumptions on the operator $A$, we do not have any detailed information on the $L^{\infty}$ norm of the eigenfunctions $\left\{\phi_{k}\right\}_{k=1}^{\infty}$ and their derivatives. Much is known when $A$ is a uniformly elliptic operator; see, e.g., [31, Ch. 5], [3, 14], and the references therein. In particular, the results from [14] imply that, when $A$ is a uniformly elliptic operator with smooth coefficients and Dirichlet or Neumann boundary conditions on some bounded domain $\Omega \subset \mathbb{R}^{d}$ with smooth boundary, then the following estimate holds:

$$
\left\|D^{n} \phi_{k}\right\|_{L^{\infty}(\Omega)} \leq C \alpha_{k}^{\frac{d-1+\frac{n}{2}}{4}}, \quad n=0,1, \ldots .
$$

We will assume that the drift $b(x)$ is Lipschitz continuous:

$$
\|b(x)-b(y)\| \leq C\|x-y\|, \quad x, y \in \mathbb{R}^{d} .
$$

Moreover, we will assume that there exist constants $C, r$ such that

$$
\left\|h_{k}\right\| \leq C\left|\alpha_{k}\right|^{r}, \quad k=1,2, \ldots
$$

Now we are ready to present the conditions that we have to impose on the spectrum of the Wiener process. First, we need to ensure the existence and uniqueness of the equations of motion (2.4a). To this end, we assume that the velocity field is sufficiently regular: ${ }^{3}$

$$
v(x, t) \in\left(C\left(\mathbb{R}^{+}, C^{1}(\Omega)\right)\right)^{d} .
$$

Assumption (2.11), together with assumption (2.9), ensures that there exists almost surely a unique solution of the equations of motion (2.4a) when the initial conditions for $(2.4 \mathrm{c})$ are distributed according to the invariant measure of this process. Furthermore, for $\gamma<2$ we have to assume conditions of the form

$$
\begin{aligned}
& \sum_{k=1}^{\infty} \sqrt{\lambda_{k}} \alpha_{k}^{\left(r+\alpha-\frac{1}{2}-\rho\right)}<\infty, \\
& \sum_{k=1}^{\infty} \sqrt{\lambda_{k}} \alpha_{k}^{\left(r+\beta-\frac{1}{2}-\rho\right)}<\infty .
\end{aligned}
$$

The specific value of the exponent $\rho$ will be given when stating our convergence theorems. For $\gamma \geq 2$, in addition to the (2.12) we further assume that

$$
\sum_{k=1}^{\infty} \sqrt{\lambda_{k}} \alpha_{k}^{r+\gamma-\frac{3}{2}}<\infty
$$

\footnotetext{
${ }^{3} \mathrm{~A}$ simple variant of $\left[25\right.$, Thm. 5.20] yields that $v(x, t) \in\left(C\left(\mathbb{R}^{+}, C^{1}(\Omega)\right)\right)^{d}$, provided that there exists a $\zeta \in(0,1)$ such that

$$
\begin{gathered}
\sum_{k=1}^{\infty} \lambda_{k} \alpha_{k}^{2(r+\alpha)-1-\zeta}<\infty \\
\sum_{k=1}^{\infty} \lambda_{k} \alpha_{k}^{2(r+\zeta \gamma)-1}<\infty
\end{gathered}
$$
}

However, these conditions are not optimal, and so we simply assume (2.11). 


$$
\begin{aligned}
& \sum_{k=1}^{\infty} \sqrt{\lambda_{k}} \alpha_{k}^{r+\delta-\frac{3}{2}}<\infty, \\
& \sum_{k=1}^{\infty} \lambda_{k} \alpha_{k}^{2 r+\beta+\gamma-2}<\infty .
\end{aligned}
$$

REMARK 2.2. Consider the case where A is a uniformly elliptic operator. From (2.8) it is easy to see that conditions (2.12) and (2.13) become

$$
\sum_{k=1}^{\infty} \sqrt{\lambda_{k}} \alpha_{k}^{(r+d / 4-5 / 8-\rho)}<\infty
$$

and

$$
\sum_{k=1}^{\infty} \lambda_{k} \alpha_{k}^{2 r+d / 2-17 / 8}<\infty
$$

respectively.

We now fix an integer $p \geq 1$. We assume that the initial conditions are random variables, independent of the $\sigma$-algebra generated by $W(t)$, with

$$
\mathbb{E}\left\|x_{0}\right\|^{2 p}<\infty, \quad \mathbb{E}\left\|y_{0}\right\|^{2 p}<\infty
$$

for $\gamma \in(0,2)$ and

$$
\mathbb{E}\left\|x_{0}\right\|^{2 p}<\infty, \quad \mathbb{E}\left\|y_{0}\right\|^{4 p}<\infty
$$

for $\gamma \in[2, \infty)$.

Now we state the convergence theorems. We start with $\gamma \in(0,2)$.

Theorem 2.3. Let $x(t)$ be the solution of (2.4a) for $\gamma \in(0,2)$. Assume that conditions (2.5), (2.6), (2.9), (2.10), (2.11), and (2.12) with $\rho=\frac{1}{2}$ and (2.14) hold. Assume further that the initial conditions for $(2.4 \mathrm{c})$ are stationary. Then $x(t)$ converges, as $\epsilon \rightarrow 0$, to $X(t)$, which satisfies the following equation:

$$
X(t)=x_{0}+\int_{0}^{t} b(X(s)) d s+\int_{0}^{t} f(X(s)) A^{-1} d W(s),
$$

the convergence being in $L^{2 p}(\Omega, C([0, T], \mathbb{R}))$ :

$$
\mathbb{E}\left(\sup _{0 \leq t \leq T}\|X(t)-x(t)\|^{2 p}\right) \leq C\left(\epsilon^{\gamma p}+\epsilon^{(2-\gamma) p-\sigma}\right),
$$

where $\sigma>0$ is arbitrarily small. The constant $C$ depends on the moments of the initial conditions, the spectrum of the Wiener process, the operator $A$, the exponent $p$, the maximum time $T$, and $\sigma$.

In order to present the convergence theorems for the case $\gamma \geq 2$ we need to introduce some notation. We denote by $\Theta, \widehat{\Theta}: \ell_{2} \rightarrow \ell_{2}$ the diagonal operators defined by

$$
\Theta=\operatorname{diag}\left\{\frac{\lambda_{j}}{2 \alpha_{j}^{2}}\right\}, \quad \widehat{\Theta}=\operatorname{diag}\left\{\frac{\lambda_{j}}{2 \alpha_{j}^{2}\left(1+\alpha_{j}\right)}\right\} .
$$


We will use the notation $\nabla \cdot A$ to denote the divergence of a matrix $A$, i.e., $\{\nabla \cdot A\}_{i}=$ $\sum_{j=1}^{d} A_{i j, j}$.

The next theorem covers the case $\gamma \in(2, \infty)$.

Theorem 2.4. Let $x(t)$ be the solution of $(2.4 \mathrm{a})$ for $\gamma \in(2, \infty)$. Assume that conditions (2.5), (2.7), (2.9), (2.10), (2.11), and (2.12a) with $\rho=\frac{1}{2},(2.12 \mathrm{~b})$ with $\rho=0,(2.13)$, and (2.15) hold. Assume further that the initial conditions for (2.4c) are stationary. Then $x(t)$ converges, as $\epsilon \rightarrow 0$, to $X(t)$, which satisfies the following equation:

$$
\begin{aligned}
X(t)= & x_{0}+\int_{0}^{t} b(X(s)) d s+\int_{0}^{t} \nabla \cdot\left(f(X(s)) \Theta f^{T}(X(s))\right) d s \\
& -\int_{0}^{t} f(X(s)) \Theta \nabla \cdot f^{T}(X(s)) d s+\int_{0}^{t} f(X(s)) A^{-1} d W(s),
\end{aligned}
$$

the convergence being in $L^{2 p}(\Omega, C([0, T], \mathbb{R}))$ :

$$
\mathbb{E}\left(\sup _{0 \leq t \leq T}\|X(t)-x(t)\|^{2 p}\right) \leq C\left(\epsilon^{2 p-\sigma}+\epsilon^{2 p(\gamma-2)-\sigma}\right),
$$

where $\sigma>0$ is arbitrarily small. The constant $C$ depends on the moments of the initial conditions, the spectrum of the Wiener process, the operator A, the exponent $p$, the maximum time $T$, and $\sigma$.

Finally, the case $\gamma=2$ is covered by the following theorem.

TheOREM 2.5. Let $x(t)$ be the solution of (2.4a) for $\gamma=2$. Assume that conditions (2.5), (2.7), (2.9), (2.10), (2.11), and (2.12a) with $\rho=\frac{1}{2},(2.12 \mathrm{~b})$ with $\rho=0$, (2.13), and (2.15) hold. Assume further that the initial conditions for (2.4c) are stationary. Then $x(t)$ converges, as $\epsilon \rightarrow 0$, to $X(t)$, which satisfies the following equation:

$$
\begin{aligned}
X(t)= & x_{0}+\int_{0}^{t} b(X(s)) d s+\int_{0}^{t} \nabla \cdot\left(f(X(s)) \widehat{\Theta} f^{T}(X(s))\right) d s \\
& -\int_{0}^{t} f(X(s)) \widehat{\Theta} \nabla \cdot f^{T}(X(s)) d s+\int_{0}^{t} f(X(s)) A^{-1} d W(s),
\end{aligned}
$$

the convergence being in $L^{2 p}(\Omega, C([0, T], \mathbb{R}))$ :

$$
\mathbb{E}\left(\sup _{0 \leq t \leq T}\|X(t)-x(t)\|^{2 p}\right) \leq C \epsilon^{2 p-\sigma}
$$

where $\sigma>0$ is arbitrarily small. The constant $C$ depends on the moments of the initial conditions, the spectrum of the Wiener process, the operator $A$, the exponent $p$, the maximum time $T$, and $\sigma$.

REMARK 2.6. The second and third integrals in (2.19) give the d-dimensional analogue of the Stratonovich correction $\frac{1}{2} f(X) f^{\prime}(X)$ in (1.6), where the system is driven by an infinite dimensional noise process. Similarly, the second and third integrals in (2.21) correspond to the drift correction in (1.7).

REMARK 2.7. The assumptions of the convergence theorems ensure Lipschitz continuity and linear growth of all terms that appear in the limiting equations, and hence the existence and uniqueness of solutions. 
REMARK 2.8. Throughout the paper we have set $\tau_{0}=1$, in order to simplify the notation. Of course, the above convergence theorems hold true for arbitrary $\tau_{0}>0$. In this case, the matrix $\widehat{\theta}$ defined in (2.18) has to be modified:

$$
\widehat{\Theta}=\operatorname{diag}\left\{\frac{\lambda_{j}}{2 \alpha_{j}^{2}\left(1+\tau_{0} \alpha_{j}\right)}\right\} .
$$

Notice that we can formally retrieve the limiting equation for $\gamma<2$ and $\gamma>2$ by sending $\tau_{0}$ in (2.23) to $\infty$ and 0 , respectively.

REMARK 2.9. Problem (2.4) for $\gamma=0$ was considered in [24]. It was shown there that, under appropriate conditions on the spectrum of the Wiener process and the operator $A$, the particle position $x(t)$ converges pathwise to the solution $X$ of a second order SDE, which we formally write as

$$
\ddot{X}=b(X)-\dot{X}+f(X) A^{-1} \dot{W} .
$$

It was proved in [24] that the convergence rate is of $\mathcal{O}\left(\epsilon^{2-\sigma}\right)$, where $\sigma>0$ is arbitrarily small. It is natural, therefore, that the convergence rate in Theorem 2.3 degenerates as $\gamma$ tends to either 0 or 2 , since the limiting equation is different in both cases.

2.3. Remarks on the convergence theorems. We now present a few comments on the convergence theorems. First, we note that the smoothness assumptions on the eigenfunctions $\left\{\phi_{k}\right\}_{k=1}^{\infty}$ are more severe for $\gamma \in[2, \infty)$ than for $\gamma \in(0,2)$. This is because, in order to prove our convergence theorems for $\gamma \geq 2$, we need additional integrations by parts, using the Itô formula. As a result, we need to assume that more moments of the particle velocity at time $t=0$ exist when $\gamma \geq 2$. Notice also that the convergence to the limiting equations becomes arbitrarily slow as $\gamma \rightarrow 0$ and $\gamma \rightarrow 2^{-}$ in Theorem 2.3, as well as $\gamma \rightarrow 2^{+}$in Theorem 2.4. This is also not surprising since the form of the coefficients in the limiting equation is discontinuous at $\gamma=2$. The extensive numerical experiments reported in [18] indicate that the convergence rates of our theorems are sharp. On the other hand, the conditions that we have to impose on the spectrum of the Wiener process, conditions (2.12) and (2.13), are not sharp and are not, in general, independent from one another. In order to optimize these conditions one needs more detailed information on the specific problem under investigation, in particular on the properties of the eigenfunctions of the operator $A$. These conditions can be optimized, for example, when $A$ is a uniformly elliptic operator; see Remarks 2.1 and 2.2 .

Let us now try to give an intuitive explanation of our results. First, for $\gamma<2$ the particle relaxation time - which is of $\mathcal{O}\left(\epsilon^{\gamma}\right)$ - is large compared to the relaxation time of the noise - which is of $\mathcal{O}\left(\epsilon^{2}\right)$ - and consequently the particles experience a rough noise with practically zero correlation time. This means that for $\gamma<2$ the OU process is not viewed from the point of view of the particle as a smooth approximation to white noise, and, as a result, the stochastic integral in the limiting equation has to be interpreted in the Itô sense. On the other hand, when $\gamma>2$, the particle relaxation time is small compared to that of the noise. Consequently, in this parameter regime the rescaled OU process is indeed a smooth Gaussian approximation to white noise, and the stochastic integral in the limiting SDE should be interpreted in the Stratonovich sense, as in (2.19), in agreement with standard theorems [2, sec. 10.3]. The case $\gamma \rightarrow \infty$ leads to the case of tracer particles whose relaxation time is zero and covered precisely by these standard theorems. 
For the case $\gamma=2$ the particle relaxation time is comparable in magnitude to the noise correlation time, and a resonance mechanism prevails, which results in the limiting stochastic integral being neither that of Itô nor that of Stratonovich. In this case the drift correction to the Itô stochastic integral depends on the detailed properties of the OU process, in particular its covariance.

It is well known that for second order SDEs the Itô and Stratonovich interpretations of the stochastic integral coincide. For certain Gaussian fields $v(x, t)$ this also happens for the limiting equations given in our convergence theorems: the Stratonovich correction, as well as its modified version from Theorem 2.5, will, in some situations, be identically zero due to the specific properties of $v(x, t)$. In this case the limiting equations are the same for all values of $\gamma$. This situation occurs, for example, in the inertial particles problem, which is discussed in section 6 , due to the fact that the fluid velocity is assumed to be homogeneous and incompressible.

Let us now outline the method that we will use in order to prove the results of this paper. The first step is to use the variation of constants formula to write the particle velocity $y(t):=\dot{x}(t)$ and particle position $x(t)$ as follows:

$$
y(t)=y_{0} e^{-\frac{t}{\epsilon \gamma}}+\epsilon^{-\gamma} \int_{0}^{t} e^{\frac{s-t}{\epsilon \gamma}} \frac{v(x(s), s)}{\epsilon} d s+\epsilon^{-\gamma} \int_{0}^{t} e^{\frac{s-t}{\epsilon \gamma}} b(x(s)) d s
$$

and

$$
\begin{aligned}
x(t)= & x_{0}+\epsilon^{\gamma} y_{0}\left(1-e^{-\frac{t}{\epsilon \gamma}}\right)+\int_{0}^{t} \frac{v(x(s), s)}{\epsilon} d s+\int_{0}^{t} b(x(s)) d s \\
& -\int_{0}^{t} e^{\frac{s-t}{\epsilon \gamma}} \frac{v(x(s), s)}{\epsilon} d s-\int_{0}^{t} e^{\frac{s-t}{\epsilon \gamma}} b(x(s)) d s,
\end{aligned}
$$

respectively. The next step is to use (2.24) and (2.25) in order to obtain sharp estimates on the moments of the particle velocity. The basic strategy will be to derive first estimates valid for $\gamma \in(0, \infty)$ and then use them in order to obtain sharper estimates valid for $\gamma \in(0,2)$. We emphasize that sharper estimates for $\gamma \in(0,2)$ are necessary for the proofs of the convergence theorems. Now, with the estimates for the moments at hand we prove that the last two integrals on the right-hand side of $(2.25)$ are small in $L^{2 p}(\Omega ; C([0, T], \mathbb{R}))$ for all values of $\gamma>0$.

Then we study the term which induces noise in (2.25), in the limit $\epsilon \rightarrow 0$, namely the first integral on the right-hand side of this equation. We refer to this as $I(t)$. We use the Itô formula, together with the estimates on the moments of the particle velocity, to show that $I(t)$ consists of an $\mathcal{O}(1)$ term plus higher order corrections. The leading order term in $I(t)$ is different for $\gamma<2, \gamma>2$, and $\gamma=2$ : this is the term which is responsible for the difference in the limiting equations for different $\gamma$. Finally, the proof of the convergence theorems is completed by an application of Gronwall's lemma.

Throughout the paper we will make extensive use of estimates on the infinite dimensional OU process $v(x, t)$, as well as the stochastic convolution

$$
\int_{0}^{t} e^{\frac{s-t}{\epsilon \gamma}} f(x(s)) A^{-1} d W(s)
$$

(Lemmas 3.2 and 3.7, respectively). The proof of Lemma 3.2 is based on Borell's inequality from the theory of Gaussian processes [1], while the proof of Lemma 3.7 uses the factorization method [25]. 
We remark that, unlike the methods used in the proofs of the convergence theorems in [24], the proof in this paper relies on the presence of the friction term $-\dot{x}$ in the equations of motion (2.1). The linear friction term enables us to obtain representations (2.24) and (2.25) for the particle velocity and position, respectively, which are necessary for analyzing the dependence of various moment bounds on $\epsilon$.

\section{Preliminary results.}

3.1. The integral formulation. The first step is to obtain an integral equation for $x(t)$ that will be more convenient for our analysis.

Lemma 3.1. Consider the equations of motion (2.4a). Then the particle position satisfies the following integral equation:

$$
\begin{aligned}
x(t)= & x_{0}+\epsilon^{\gamma} y_{0}\left(1-e^{-\frac{t}{\epsilon \gamma}}\right)+\int_{0}^{t}\left(1-e^{\frac{s-t}{\epsilon \gamma}}\right) \frac{v(x(s), s)}{\epsilon} d s \\
& +\int_{0}^{t}\left(1-e^{\frac{s-t}{\epsilon \gamma}}\right) b(x(s)) d s .
\end{aligned}
$$

Proof. We start by solving the equation for $y(t)=\dot{x}(t)$ using the variation of constants formula:

$$
y(t)=y_{0} e^{-\frac{t}{\epsilon \gamma}}+\epsilon^{-\gamma} \int_{0}^{t} e^{\frac{s-t}{\epsilon \gamma}} \frac{v(x(s), s)}{\epsilon} d s+\epsilon^{-\gamma} \int_{0}^{t} e^{\frac{s-t}{\epsilon \gamma}} b(x(s)) d s .
$$

Another integration will give us an integral equation for $x(t)$ which involves a double integral:

$$
\begin{aligned}
x(t) & =x_{0}+\int_{0}^{t} y_{0} e^{-\frac{s}{\epsilon \gamma}} d s+\frac{1}{\epsilon^{\gamma}} \int_{0}^{t}\left(\int_{0}^{\ell} e^{\frac{s-\ell}{\epsilon \gamma}} H(x(s), s) d s\right) d \ell \\
& =x_{0}+\epsilon^{\gamma} y_{0}\left(1-e^{-\frac{t}{\epsilon \gamma}}\right)+I(t),
\end{aligned}
$$

where

$$
H(s)=\frac{v(x(s), s)}{\epsilon}+b(x(s)) .
$$

We can reduce $I(t)$ to a single integral as follows: First, we define the following function:

$$
F(\ell)=\int_{0}^{\ell} e^{\frac{s}{\epsilon \gamma}} H(s) d s .
$$

Now we perform an integration by parts:

$$
\begin{aligned}
I(t) & =\frac{1}{\epsilon^{\gamma}} \int_{0}^{t}\left(\int_{0}^{\ell} e^{\frac{s-\ell}{\epsilon \gamma}}\left(\frac{v(x(s), s)}{\epsilon}+b(s)\right) d s\right) d \ell \\
& =\frac{1}{\epsilon^{\gamma}} \int_{0}^{t} e^{-\frac{\ell}{\epsilon \gamma}} F(\ell) d \ell \\
& =-\left.F(\ell) e^{-\frac{\ell}{\epsilon} \gamma}\right|_{\ell=0} ^{\ell=t}+\int_{0}^{t} e^{-\frac{\ell}{\epsilon \gamma}} d F(\ell) \\
& =-e^{-\frac{t}{\epsilon \gamma}} \int_{0}^{t} e^{\frac{s}{\epsilon \gamma}} H(s) d s+\int_{0}^{t} H(s) d s \\
& =\int_{0}^{t}\left(1-e^{\frac{s-t}{\epsilon \gamma}}\right) \frac{v(x(s), s)}{\epsilon} d s+\int_{0}^{t}\left(1-e^{\frac{s-t}{\epsilon \gamma}}\right) b(x(s)) d s .
\end{aligned}
$$


Substituting (3.4) into (3.3) we obtain (3.1).

Throughout the paper we will use the following notation:

$$
\begin{gathered}
I_{1}(t)=\epsilon^{\gamma} y_{0}\left(1-e^{-\frac{t}{\epsilon \gamma}}\right), \\
I_{2}(t)=\int_{0}^{t} \frac{f(x(s)) \eta(s)}{\epsilon} d s \\
I_{3}(t)=-\int_{0}^{t} e^{\frac{s-t}{\epsilon \gamma}} \frac{f(x(s)) \eta(s)}{\epsilon} d s \\
I_{4}(t)=-\int_{0}^{t} e^{\frac{s-t}{\epsilon \gamma}} b(x(s)) d s .
\end{gathered}
$$

Using this notation the particle position $x(t)$ can be written in the form

$$
x(t)=x_{0}+\sum_{i=1}^{4} I_{i}(t)+\int_{0}^{t} b(x(s)) d s .
$$

We clearly have

$$
\mathbb{E}\left(\sup _{0 \leq t \leq T}\left\|I_{1}(t)\right\|^{2 p}\right) \leq C \epsilon^{2 \gamma p}
$$

Now we want to study terms $I_{2}(t), I_{3}(t)$, and $I_{4}(t)$. As explained in section 2.3 , we want to show that $I_{3}(t)$ and $I_{4}(t)$ are $o(1)$ in $L^{2 p}(\Omega, C([0, T], \mathbb{R}))$ for every $\gamma \in(0, \infty)$ and then show that the behavior of the term $I_{2}(t)$ as $\epsilon \rightarrow 0$ depends on $\gamma$. In order to obtain the necessary bounds we will need sharp estimates on the moments of the particle velocity. We will obtain these estimates in section 3.3. Before doing this, we need some estimates on the velocity field $v(x, t)$.

3.2. Estimates on the colored noise. In this subsection we present two results which will be used in the proofs of the convergence theorems. We start with an estimate on the infinite dimensional OU process.

LEMma 3.2. Assume that conditions (2.7), (2.10), and (2.12a) with $\rho \in \mathbb{R}$ are satisfied. Then the following estimate holds:

$$
\mathbb{E}\left(\sup _{0 \leq t \leq T}\left\|A^{-\rho} v(x(t), t)\right\|^{2 p}\right) \leq C \epsilon^{-\sigma},
$$

where $\sigma>0$ is arbitrarily small.

Proof. Let $\eta(t)=\left\{\eta_{k}(t)\right\}_{k=1}^{\infty}: \ell_{2} \rightarrow \mathbb{R}$ be the solution of (2.3) with stationary initial conditions. The $k$ th component $\eta_{k}(t)$ solves the equation

$$
d \eta_{k}=-\alpha_{k} \eta_{k} d t+\sqrt{\lambda_{k}} d W_{k} .
$$

A simple variant of Theorem A.1 from [24] yields

$$
\mathbb{E}\left(\sup _{0 \leq t \leq T}\left|\eta_{k}(t)\right|^{2 p}\right) \leq\left(\frac{\lambda_{k}}{\alpha_{k}}\right)^{2 p}\left(1+\ln \left(\frac{\alpha_{k} T}{\epsilon^{2}}\right)\right) .
$$


Let $Y(x(t), t):=A^{-\rho} v(x(t), t)$. We first consider the case $p=1$. We have

$$
\begin{aligned}
& \mathbb{E}\left(\sup _{0 \leq t \leq T}\|Y(x(t), t)\|^{2}\right) \\
&=\mathbb{E}\left(\sup _{0 \leq t \leq T}\left\|\sum_{k=1}^{\infty} h_{k} \phi_{k}(x(t)) \alpha_{k}^{-\rho} \eta_{k}(t)\right\|^{2}\right) \\
&=\mathbb{E}\left(\sup _{0 \leq t \leq T}\left\|\sum_{k, \ell=1}^{\infty} h_{k} \cdot h_{\ell} \phi_{k}(x(t)) \phi_{\ell}(x(t)) \alpha_{k}^{-\rho} \alpha_{\ell}^{-\rho} \eta_{k}(t) \eta_{\ell}(t)\right\|\right) \\
& \leq \mathbb{E}\left(\sup _{0 \leq t \leq T}\left(\sum_{k^{+}}\left\|h_{k}\right\|^{2}\left|\phi_{k}(x(t))\right|^{2} \alpha_{k}^{-2 \rho}\left|\eta_{k}(t)\right|^{2}\right)\right) \\
&+\mathbb{E}\left(\sup _{0 \leq t \leq T}\left(\sum_{k=1}^{\infty} \sum_{\ell \neq k}\left\|h_{k}\right\|\left\|h_{\ell}\right\| \phi_{k}(x(t)) \phi_{\ell}(x(t)) \alpha_{k}^{-\rho} \alpha_{\ell}^{-\rho} \eta_{k}(t) \eta_{\ell}(t)\right)\right) \\
& \leq C \sum_{k=1}^{\infty} \frac{|k|^{2(r+\alpha)}}{\alpha_{k}^{2 \rho}} \mathbb{E}\left(\sup _{0 \leq t \leq T}\left|\eta_{k}(t)\right|^{2}\right)+C\left(\sum_{k=1}^{\infty} \frac{|k|^{r+\alpha}}{\alpha_{k}^{\rho}} \mathbb{E}\left(\sup _{0 \leq t \leq T}\left|\eta_{k}(t)\right|\right)\right)^{2} \\
& \leq C\left(\sum_{k=1}^{\infty} \frac{\lambda_{k}|k|^{2(r+\alpha)}}{\alpha_{k}^{1+2 \rho}}\right) \epsilon^{-\sigma}+C\left(\sum_{k=1}^{\infty} \frac{\sqrt{\lambda_{k}}|k|^{r+\alpha}}{\alpha_{k}^{\frac{1}{2}+\rho}}\right) \epsilon^{-\sigma} \\
& \leq C \epsilon^{-\sigma},
\end{aligned}
$$

on account of condition (2.12a). We can proceed in the same way for $p>1$, by breaking the sums into various parts, until we have sums that involve independent OU processes. Condition (2.12a) ensures the summability of all the sums that appear. The lemma is proved.

Using the above lemma we can easily obtain the following estimate.

Lemma 3.3. Assume that the conditions of Lemma 3.2 hold. Define

$$
I(t)=\int_{0}^{t} e^{\frac{s-t}{\epsilon \gamma}} A^{-\rho} v(x(s), s) d s .
$$

Then the following estimate holds:

$$
\mathbb{E}\left(\sup _{0 \leq t \leq T}\|I(t)\|^{2 p}\right) \leq C \epsilon^{2 p \gamma-\sigma},
$$

where $\sigma>0$ is arbitrarily small.

Proof. We have, for $t \in[0, T]$,

$$
\begin{aligned}
\|I(t)\|^{2 p} & =\left\|\int_{0}^{t} e^{\frac{s-t}{\epsilon \gamma}} A^{-\rho} v(x(s), s) d s\right\|^{2 p} \\
& \leq \sup _{0 \leq s \leq T}\left\|A^{-\rho} v(x(s), s)\right\|^{2 p}\left(\int_{0}^{t} e^{\frac{s-t}{\epsilon \gamma}} d s\right)^{2 p} \\
& \leq \epsilon^{2 p \gamma} \sup _{0 \leq t \leq T}\left\|A^{-\rho} v(x(s), s)\right\|^{2 p} .
\end{aligned}
$$

Lemma 3.2 now yields estimate (3.11). 
REMARK 3.4. The techniques used in the proof of Lemma 3.2 enable us to conclude that we can bound uniformly all moments of the field $v(x, t)$ :

$$
\mathbb{E}\left\|A^{-\rho} v(x, t)\right\|^{2 p} \leq C,
$$

provided that the assumptions of the lemma are satisfied. Furthermore, the method of proof of Lemma 3.3 gives

$$
\mathbb{E}\|I(t)\|^{2 p} \leq C \epsilon^{2 p \gamma},
$$

where $I(t)$ is defined in (3.10).

REMARK 3.5. Lemma 3.3 with $\rho=0$ provides us with estimates for $I_{3}(t)$ and $I_{4}(t)$ which we will use for $\gamma \in[2, \infty)$ :

$$
\mathbb{E}\left(\sup _{0 \leq t \leq T}\left\|I_{3}(t)\right\|^{2 p}\right) \leq C \epsilon^{2 p(\gamma-1)-\sigma}, \quad \gamma \in[2, \infty)
$$

and

$$
\mathbb{E}\left(\sup _{0 \leq t \leq T}\left\|I_{2}(t)\right\|^{2 p}\right) \leq C \epsilon^{-2 p-\sigma}, \quad \gamma \in[2, \infty),
$$

where $\sigma>0$ is arbitrarily small. This estimate is not sharp enough when $\gamma \in(0,2)$, and we need to improve it. This will be accomplished in Corollary 3.13.

REMARK 3.6. Assume that the moments of the particle velocity $y(t)$ satisfy

$$
\mathbb{E}\left(\sup _{0 \leq t \leq T}\|y(t)\|^{2 p}\right) \leq C \epsilon^{\zeta p}
$$

for some $\zeta \in \mathbb{R}$. A repeated use of Hölder's inequality, together with the Gaussianity of the process $\eta(t)$ as in the proof of Lemma 4.3 in [24], enables us to prove that

$$
\mathbb{E}\left(\sup _{0 \leq t \leq T}\|y(t)\|^{2 p}\|\eta(t)\|_{\ell^{2}}^{2 n}\right) \leq \epsilon^{-\zeta p-\sigma}
$$

for every $n$, assuming that $\operatorname{Tr}(Q)<\infty$, with $\sigma>0$ arbitrarily small. In what follows we will have the occasion to use estimate (3.13) and variants of it repeatedly.

We proceed now with an estimate on a stochastic integral.

Lemma 3.7. Consider the stochastic integral

$$
I(t)=\int_{0}^{t} e^{\frac{s-t}{\epsilon \tau}} f(x(s)) A^{-1} d W(s) .
$$

Assume that conditions (2.6), (2.10), and (2.12a) with $\rho=\frac{1}{2}$ hold. Then we have the following estimate:

$$
\mathbb{E}\left(\sup _{0 \leq t \leq T}\|I(t)\|^{2 p}\right) \leq C \epsilon^{p \gamma-\sigma}
$$

where $\sigma>0$ is arbitrarily small.

Proof. We fix $\alpha \in\left(0, \frac{1}{2}\right)$ and use the factorization method from [25, sec. 5.3] to obtain

$$
\begin{aligned}
I(t) & :=\int_{0}^{t} e^{\frac{s-t}{\epsilon \gamma}} f(x(s)) A^{-1} d W(s) \\
& =\frac{\sin (\pi \alpha)}{\pi} \int_{0}^{t} e^{\frac{s-t}{\epsilon \gamma}}(t-s)^{\alpha-1} Y(s) d s
\end{aligned}
$$


where

$$
Y(s)=\int_{0}^{s} e^{\frac{\sigma-s}{\epsilon \gamma}}(s-\sigma)^{-\alpha} f(x(\sigma)) A^{-1} d W(\sigma) .
$$

We choose $m>\frac{1}{2 \alpha}$ and use the Hölder inequality to obtain

$$
\|I(t)\|^{2 m} \leq C\left(\int_{0}^{t}\left|e^{\frac{s-t}{\epsilon \gamma}}(t-s)^{\alpha-1}\right|^{\frac{2 m}{2 m-1}} d s\right)^{2 m-1} \int_{0}^{t}\|Y(s)\|^{2 m} d s .
$$

A change of variables now yields

$$
\begin{aligned}
J(t) & :=\int_{0}^{t}\left|e^{\frac{s-t}{\epsilon \gamma}}(t-s)^{\alpha-1}\right|^{\frac{2 m}{2 m-1}} d s \\
& =\left(\frac{2 m-1}{2 m}\right)^{\frac{2 m-1}{2 m}(\alpha+2(m-1))} \epsilon^{\gamma \frac{2 m \alpha-1}{2 m-1}} \int_{0}^{t \frac{2 m}{2 m-1} \epsilon^{-\gamma}} e^{-z} z^{\frac{2 m}{2 m-1}(\alpha-1)} d z \\
& \leq \epsilon^{\gamma \frac{2 m \alpha-1}{2 m-1}} \int_{0}^{\infty} e^{-z} z^{\frac{2 m}{2 m-1}(\alpha-1)} d z \\
& \leq C \epsilon^{\frac{\gamma 2 m \alpha-1}{2 m-1}} .
\end{aligned}
$$

In the above estimate we used the fact that, since $m>\frac{1}{2 \alpha}$, we have $e^{-z} z^{\frac{2 m}{2 m-1}(\alpha-1)} \in$ $L^{1}\left(\mathbb{R}^{+}\right)$. Consequently, we have

$$
\mathbb{E}\left(\sup _{0 \leq t \leq T}\|I(t)\|^{2 m}\right) \leq C \epsilon^{\gamma(2 m \alpha-1)} \mathbb{E} \int_{0}^{T}\|Y(s)\|^{2 m} d s .
$$

To proceed further, we use [25, Lem. 7.2] to deduce that there exists a constant $C_{m}>0$ depending only on $m$ such that

$$
\sup _{0 \leq s \leq T} \mathbb{E}\|Y(s)\|^{2 m} \leq C_{m} \mathbb{E}\left(\int_{0}^{s} e^{-2 \frac{s-\sigma}{\epsilon \mathcal{\gamma}}}(s-\sigma)^{-2 \alpha}\left\|f(x(\sigma)) A^{-1}\right\|_{L_{2}^{0}}^{2} d \sigma\right)^{m},
$$

with

$$
\begin{aligned}
\left\|f(x(\sigma)) A^{-1}\right\|_{L_{2}^{0}}^{2} & :=\operatorname{Tr}\left[\left(f(x(\sigma)) A^{-1}\right) Q\left(f(x(\sigma)) A^{-1}\right)^{*}\right] \\
& =\sum_{k=1}^{\infty} \frac{\lambda_{k}\left|\phi_{k}(x(\sigma))\right|^{2}\left\|h_{k}\right\|^{2}}{\alpha_{k}^{2}} \\
& \leq C \sum_{k=1}^{\infty} \lambda_{k}\left|\alpha_{k}\right|^{2(\alpha+r-1)}<\infty
\end{aligned}
$$

on account of condition (2.12a) with $\rho=\frac{1}{2}$. Now we can apply the same change of variables that we used in the estimate for $J(t)$ to obtain

$$
\begin{aligned}
\sup _{0 \leq s \leq T} \mathbb{E}\|Y(s)\|^{2 m} & \leq C \mathbb{E}\left(\int_{0}^{s} e^{2 \frac{\sigma-s}{\epsilon \gamma}}(s-\sigma)^{-2 \alpha} d \sigma\right)^{m} \\
& \leq C\left(\epsilon^{\gamma(1-2 \alpha)} \int_{0}^{\frac{2 s}{\epsilon \gamma}} e^{-z} z^{-2 \alpha} d z\right)^{m} \\
& \leq C \epsilon^{\gamma m(1-2 \alpha)} .
\end{aligned}
$$


From the above estimates we conclude that

$$
\mathbb{E}\left(\sup _{0 \leq t \leq T}\|I(t)\|^{2 m}\right) \leq C \epsilon^{\gamma(m-1)} .
$$

Now estimate (3.14) follows for $p>\frac{1}{2 \alpha}$ upon taking $p=m$. For $p \leq \frac{1}{2 \alpha}$ we apply the Hölder inequality to obtain

$$
\mathbb{E}\left(\sup _{0 \leq t \leq T}\|I(t)\|^{2 p}\right) \leq\left(\mathbb{E}\left(\sup _{0 \leq t \leq T}\|I(t)\|^{2 m}\right)\right)^{\frac{p}{m}} \leq C \epsilon^{\gamma p-\frac{p}{m}},
$$

which completes the proof of the lemma, since $m$ can be chosen to be arbitrarily large.

3.3. Bounds on the moments of $y(t)$. In this subsection we will obtain bounds on the moments of $y(t)$ that we will need for the convergence theorem. In order to obtain estimates on the moments of the particle velocity we first need to obtain a crude estimate on the moments of the particle position. This estimate will be improved later.

LEMma 3.8. Let $x(t)$ satisfy (2.4a). Assume that (2.9) and (2.14) as well as the conditions of Lemma 3.2 with $\rho=0$ are satisfied. Then the following estimate holds:

$$
\mathbb{E}\left(\sup _{0 \leq t \leq T}\|x(t)\|^{2 p}\right) \leq C \epsilon^{-2 p-\sigma},
$$

where $\sigma>0$ is arbitrarily small.

Proof. The particle position is given by (3.1), which can be written in the form

$$
x(t)=x_{0}+I_{1}(t)+I_{2}(t)+I_{3}(t)+\int_{0}^{t}\left(1-e^{\frac{t-s}{\epsilon \gamma}}\right) b(x(s)) d s .
$$

The Lipschitz continuity, assumption (2.9), of $b(x)$ implies that there exists $C>0$ such that

$$
\|b(x)\| \leq C(1+\|x\|) .
$$

We use this, together with estimates (3.7) and (3.12), as well as Lemma 3.2, to obtain

$$
\begin{aligned}
\mathbb{E}\left(\sup _{0 \leq t \leq T}\|x(t)\|^{2 p}\right) \leq & \mathbb{E}\left\|x_{0}\right\|^{2 p}+C \epsilon^{2 \gamma p}+C \epsilon^{-2 p-\sigma}+C \epsilon^{2 p(\gamma-1)-\sigma} \\
& +C\left(1+\int_{0}^{T} \mathbb{E}\left(\sup _{0 \leq t \leq T}\|x(t)\|^{2 p}\right) d t\right) \\
\leq & C \epsilon^{-2 p-\sigma}+\int_{0}^{T} \mathbb{E}\left(\sup _{0 \leq t \leq T}\|x(t)\|^{2 p}\right) d t .
\end{aligned}
$$

Estimate (3.16) now follows from Gronwall's lemma.

We have already mentioned that the bounds on the moments of the particle velocity will be different for $\gamma<2$ and $\gamma \geq 2$. We start with the regime $\gamma \in[2, \infty)$.

Lemma 3.9. Let $x(t)$ satisfy $(2.4 \mathrm{a})$, and let $y(t)=\dot{x}(t)$. Assume that assumptions (2.9) and (2.14) as well as the conditions of Lemma 3.2 with $\rho=0$ are satisfied. Then the following estimate holds:

$$
\mathbb{E}\left(\sup _{0 \leq t \leq T}\|y(t)\|^{2 p}\right) \leq C \epsilon^{-2 p-\sigma},
$$

where $\sigma>0$ is arbitrarily small. 
Proof. The particle velocity is given by (3.2), which can be written in the form

$$
y(t)=y_{0} e^{-\frac{t}{\epsilon}}-\epsilon^{-\gamma} I_{3}(t)-\epsilon^{-\gamma} I_{4}(t) .
$$

Lemma 3.8 and estimate (3.17) give

$$
\mathbb{E}\left(\sup _{0 \leq t \leq T}\left\|I_{4}(t)\right\|^{2 p}\right) \leq C \epsilon^{2 p(\gamma-1)-\sigma} .
$$

We use the above estimate, together with (3.12a), to obtain

$$
\begin{aligned}
\mathbb{E}\left(\sup _{0 \leq t \leq T}\|y(t)\|^{2 p}\right) & \leq C_{1}+C_{2} \epsilon^{-2 \gamma p} \mathbb{E}\left(\sup _{0 \leq t \leq T}\left\|I_{3}(t)\right\|^{2 p}\right)+C_{3} \epsilon^{-2 \gamma p} \mathbb{E}\left(\sup _{0 \leq t \leq T}\left\|I_{4}(t)\right\|^{2 p}\right) \\
& \leq C\left(\epsilon^{-2 p-\sigma}+1\right),
\end{aligned}
$$

from which the estimate follows.

Estimate (3.18) will be sufficient for our purposes for $\gamma \in[2, \infty)$. However, it is not sharp enough for $\gamma \in(0,2)$. In order to prove the convergence theorem for values of $\gamma$ in this parameter regime, we need to improve the estimate for the $2 p$ th moments of the particle position and particle velocity. For these two estimates we need some preliminary estimates which will also be used in the proof of the convergence theorem. We start with the following lemma.

LEMma 3.10. Assume that conditions (2.5), (2.6), and (2.10) together with (2.12b) with $\rho \in \mathbb{R}$ hold. Fix $x, y \in \mathbb{R}^{d}$, and define $d f(x) y \in L\left(\ell_{2}, \mathbb{R}^{d}\right)$ by

$$
\{(d f) y\} \gamma=\sum_{k=1}^{\infty} h_{k} y \cdot \nabla \phi_{k} \gamma_{k} .
$$

Let

$$
I(t)=\int_{0}^{t} e^{\frac{s-t}{\epsilon \tau}} d f(x(s)) y(s) A^{-\rho} \eta(s) d s
$$

and

$$
\widehat{I}(t)=\int_{0}^{t} d f(x(s)) y(s) A^{-\rho} \eta(s) d s .
$$

Then the following estimates hold:

$$
\mathbb{E}\left(\sup _{0 \leq t \leq T}\|I(t)\|^{2 p}\right) \leq C \epsilon^{(2 p-1) \gamma-2 p-\sigma}
$$

and

$$
\mathbb{E}\left(\sup _{0 \leq t \leq T}\|\widehat{I}(t)\|^{2 p}\right) \leq C \epsilon^{-2 p-\sigma}
$$

where $\sigma>0$ is arbitrarily small.

Proof. An application of the Hölder inequality yields

$$
\begin{aligned}
\|I(t)\|^{2 p} & \leq\left(\int_{0}^{t} e^{\left(\frac{s-t}{\epsilon \gamma} \frac{2 p}{2 p-1}\right)} d s\right)^{2 p-1} \int_{0}^{t}\left\|d f(x(s)) y(s) A^{-\rho} \eta(s)\right\|^{2 p} d s \\
& \leq \epsilon^{(2 p-1) \gamma} \int_{0}^{t}\left\|d f(x(s)) y(s) A^{-\rho} \eta(s)\right\|^{2 p} d s .
\end{aligned}
$$


A simple variant of (3.13), together with calculations similar to those used in the proof of Lemma 3.2, gives

$$
\begin{aligned}
\mathbb{E}\left(\sup _{0 \leq t \leq T}\|I(t)\|^{2 p}\right) & \leq \epsilon^{(2 p-1) \gamma} \int_{0}^{T} \mathbb{E}\left\|d f(x(s)) y(s) A^{-\rho} \eta(s)\right\|^{2 p} d s \\
& \leq C \epsilon^{(2 p-1) \gamma} \int_{0}^{T} \mathbb{E}\left(\sum_{i=1}^{\infty}\left|\sum_{j=1}^{\infty} \sum_{k=1}^{d} f_{i j, k}(x(s)) y_{k}(s) \alpha_{j}^{-\rho} \eta_{j}(s)\right|^{2}\right)^{p} d s \\
& \leq C \epsilon^{(2 p-1) \gamma} \int_{0}^{T} \mathbb{E}\left(\left(\sum_{j=1}^{\infty} \alpha_{k}^{\beta+r-\rho} \eta_{j}(s)\right)^{2 p}\|y(s)\|^{2 p}\right) \\
& \leq C \epsilon^{(2 p-1) \gamma-2 p-\sigma}
\end{aligned}
$$

assuming that condition (2.12b) holds. This proves (3.20). The proof of (3.21) is almost identical and is omitted.

We introduce some notation that we will use repeatedly throughout the rest of the paper. We set

$$
\begin{array}{lll}
J_{1}(t):=\left[A^{-1} v(x(t), t)-e^{-\frac{t}{\epsilon \gamma}} v\left(x_{0}, 0\right)\right], & J_{2}(t):=\int_{0}^{t} e^{\frac{s-t}{\epsilon \gamma}} d f(x(s)) y(s) A^{-1} \eta(s) d s, \\
J_{3}(t):=\int_{0}^{t} e^{\frac{s-t}{\epsilon \gamma}} f(x(s)) A^{-1} d W(s), & J_{4}(t):=\int_{0}^{t} e^{\frac{s-t}{\epsilon \gamma}} A^{-1} v(x(s), s) d s .
\end{array}
$$

Lemmas 3.2, 3.3, 3.7, and 3.10, assuming that the initial conditions for $v(x, t)$ are stationary and that conditions (2.12a) with $\rho=\frac{1}{2}$ and (2.12b) with $\rho=1$ are satisfied, provide us with the following bounds:

$$
\begin{array}{ll}
\mathbb{E}\left(\sup _{0 \leq t \leq T}\left\|J_{1}(t)\right\|^{2 p}\right) \leq C \epsilon^{-\sigma}, & \mathbb{E}\left(\sup _{0 \leq t \leq T}\left\|J_{2}(t)\right\|^{2 p}\right) \leq C \epsilon^{(2 p-1) \gamma-2 p-\sigma}, \\
\mathbb{E}\left(\sup _{0 \leq t \leq T}\left\|J_{3}(t)\right\|^{2 p}\right) \leq C \epsilon^{p \gamma-\sigma}, & \mathbb{E}\left(\sup _{0 \leq t \leq T}\left\|J_{4}(t)\right\|^{2 p}\right) \leq C \epsilon^{2 p \gamma-\sigma} .
\end{array}
$$

Lemma 3.11. Let $x(t)$ be given by (3.1), and let $y(t)=\dot{x}(t)$ be given by (3.2). Suppose that conditions (2.9) and (2.12) with $\rho=1$ hold. Then for $\gamma \in(0,2)$ the following estimate holds:

$$
\mathbb{E}\left(\sup _{0 \leq t \leq T}\|x(t)\|^{2 p}\right) \leq C
$$

Proof. Consider first the term $I_{2}(t)$ defined in $(3.5 \mathrm{~b})$. We integrate by parts to obtain

$$
I_{2}(t)=\int_{0}^{t} f(x(s)) A^{-1} d W(s)-\epsilon \widehat{J}_{1}(t)+\epsilon \widehat{J}_{2}(t)
$$

where

$$
\widehat{J}_{1}(t):=\left[A^{-1} v(x(t), t)-A^{-1} v(x(0), 0)\right]
$$


and

$$
\widehat{J}_{2}(t)=\int_{0}^{t} d f(x(s)) y(s) A^{-1} \eta(s) d s .
$$

Lemma 3.2 with $\rho=1$ gives

$$
\mathbb{E}\left(\sup _{0 \leq t \leq T}\left\|\epsilon \widehat{J}_{1}(t)\right\|^{2 p}\right) \leq C \epsilon^{2 p-\sigma}
$$

Moreover, Lemma 3.10 with $\rho=1$, in particular estimate (3.21), yields

$$
\mathbb{E}\left(\sup _{0 \leq t \leq T}\left\|\epsilon \widehat{J}_{2}(t)\right\|^{2 p}\right) \leq C
$$

Furthermore, the Burkholder-Davis-Gundy inequality, assuming that condition (2.12a) with $\rho=\frac{1}{2}$ holds, yields

$$
\mathbb{E}\left(\sup _{0 \leq t \leq T}\left\|\int_{0}^{t} f(x(s)) A^{-1} d W(s)\right\|^{2 p}\right) \leq C .
$$

We put the above estimates together to conclude that

$$
\mathbb{E}\left(\sup _{0 \leq t \leq T}\left\|I_{2}(t)\right\|^{2 p}\right) \leq C .
$$

We use this estimate, together with (3.12a) and the assumptions (2.14) in (3.1), to obtain

$$
\begin{aligned}
\mathbb{E}\left(\sup _{0 \leq t \leq T}\|x(t)\|^{2 p}\right) \leq & C \mathbb{E}\left\|x_{0}\right\|^{2 p}+C \mathbb{E}\left\|y_{0}\right\|^{2 p}+C \epsilon^{2 p(\gamma-1)-\sigma} \\
& +C \int_{0}^{T} \mathbb{E}\left(\sup _{0 \leq t \leq T}\|x(t)\|^{2}\right) d t \\
\leq & C+\int_{0}^{T} \mathbb{E}\left(\sup _{0 \leq t \leq T}\|x(t)\|^{2}\right) d t .
\end{aligned}
$$

Estimate (3.23) now follows from Gronwall's lemma.

Now we are ready to obtain a sharp bound on the moments of the particle velocity for $\gamma \in(0,2)$.

Lemma 3.12. Let $x(t)$ be the solution of $(2.4 \mathrm{a})$, and let $y(t)=\dot{x}(t)$. Assume that $\mathbb{E}\left\|y_{0}\right\|^{2 p}<\infty$ and that conditions (2.6), (2.9), (2.10), and (2.12a) with $\rho=\frac{1}{2}$ and (2.12b) with $\rho=1$ are satisfied. Then the following estimate holds:

$$
\mathbb{E}\left(\sup _{0 \leq t \leq T}\|y(t)\|^{2 p}\right) \leq C \epsilon^{-\gamma p-\sigma}, \quad \gamma \in(0,2) .
$$

Proof. Lemma 3.11 and estimate (3.17) give

$$
\mathbb{E}\left(\sup _{0 \leq t \leq T}\left\|I_{4}(t)\right\|^{2 p}\right) \leq C \epsilon^{2 p \gamma}
$$


We combine this estimate with (3.2) to deduce

$$
\mathbb{E}\left(\sup _{0 \leq t \leq T}\|y(t)\|^{2 p}\right) \leq C_{1}+C_{2} \epsilon^{-2 p \gamma} \mathbb{E}\left(\sup _{0 \leq t \leq T}\left\|I_{3}(t)\right\|^{2 p}\right) .
$$

We need to get a sharper estimate on $I_{3}(t)$ than (3.12a). For this we need to integrate by parts. We apply Itô's formula to the function

$$
G_{i k}(s, x, \eta)=e^{\frac{s}{\epsilon^{\gamma}}} f_{i k}(x(s)) \frac{\eta_{k}(s)}{\alpha_{k}}
$$

to obtain, after some algebra,

$$
\begin{aligned}
I_{3}(t)= & \epsilon\left[A^{-1} v(x(t), t)-e^{-\frac{t}{\epsilon \gamma}} A^{-1} v\left(x_{0}, 0\right)\right] \\
& -\epsilon \int_{0}^{t} e^{\frac{s-t}{\epsilon \gamma}} d f(x(s)) y(s) A^{-1} \eta(s) d s \\
& -\int_{0}^{t} e^{\frac{s-t}{\epsilon \gamma}} f(x(s)) A^{-1} d W(s) \\
& +\epsilon^{1-\gamma} \int_{0}^{t} e^{\frac{s-t}{\epsilon \gamma}} A^{-1} v(x(s), s) d s \\
= & \epsilon J_{1}(t)-\epsilon J_{2}(t)-J_{3}(t)+\epsilon^{1-\gamma} J_{4}(t) .
\end{aligned}
$$

Consequently, on account of estimates (3.22),

$$
\mathbb{E}\left(\sup _{0 \leq t \leq T}\left\|I_{3}(t)\right\|^{2 p}\right) \leq C\left(\epsilon^{2 p-\sigma}+\epsilon^{(2 p-1) \gamma-\sigma}+\epsilon^{p \gamma-\sigma}\right) .
$$

Thus

$$
\mathbb{E}\left(\sup _{0 \leq t \leq T}\|y(t)\|^{2 p}\right) \leq C\left(\epsilon^{2 p(1-\gamma)-\sigma}+\epsilon^{-\gamma-\sigma}+\epsilon^{-p \gamma-\sigma}\right),
$$

from which estimate (3.25) follows upon noticing that, for $\gamma \in(0,2), 2 p(1-\gamma)>-p \gamma$, as well as that $p \geq 1 .{ }^{4}$ The proof of the lemma is now complete.

From the above lemma we can obtain sharper bounds on $I_{3}(t)$ and $\widehat{J}_{2}(t)$ defined in (3.19) for $\gamma \in(0,2)$.

Corollary 3.13. Let $x(t)$ be the solution of $(2.4 \mathrm{a})$. Suppose that the conditions of Lemma 3.12 hold and that $\gamma \in(0,2)$. Then $I_{3}(t)$ and $\widehat{J}_{2}(t)$ satisfy the following estimates:

$$
\mathbb{E}\left(\sup _{0 \leq t \leq T}\left\|I_{3}(t)\right\|^{2 p}\right) \leq C \epsilon^{\gamma p-\sigma}, \quad \gamma \in(0,2)
$$

and

$$
\mathbb{E}\left(\sup _{0 \leq t \leq T}\left\|\widehat{J}_{2}(t)\right\|^{2 p}\right) \leq C \epsilon^{-\gamma p-\sigma}, \quad \gamma \in(0,2),
$$

respectively, where $\sigma>0$ is arbitrarily small.

\footnotetext{
${ }^{4}$ The presence of the term $C \epsilon^{2-2 \gamma}$ in the bound of the second moment of the particle velocity can become important when studying the problem considered in this paper numerically. We refer the reader to [18] for details.
} 
Proof. Consider the term $J_{2}(t)$ defined in (3.27). Lemmas 3.10 and 3.12 imply that for $\gamma \in(0,2)$ we have

$$
\begin{aligned}
\mathbb{E}\left(\sup _{0 \leq t \leq T}\left\|J_{2}(t)\right\|^{2 p}\right) & \leq C\left(\epsilon^{(p-1) \gamma-\sigma}+\epsilon^{2(p-1) \gamma-\sigma}\right) \\
& \leq C \epsilon^{(p-1) \gamma-\sigma},
\end{aligned}
$$

since $p \geq 1$. We use now (3.27) and the above estimate, together with (3.22), to obtain

$$
\begin{aligned}
\mathbb{E}\left(\sup _{0 \leq t \leq T}\left\|I_{3}(t)\right\|^{2 p}\right) & \leq C\left(\epsilon^{2 p-\sigma}+\epsilon^{2 p-+(p-1) \gamma-\sigma}+\epsilon^{p \gamma-\sigma}\right) \\
& \leq C \epsilon^{p \gamma-\sigma}
\end{aligned}
$$

where we have used the facts that $p \geq 1$ and $\gamma<2$. Calculations similar to the ones used in the proof of Lemma 3.10, together with estimate (3.25), yield estimate (3.29).

4. Drift corrections to the Itô integral. Estimates (3.7), (3.26), (3.12a), and (3.28), together with (3.6), imply that $x(t)$ is of the form

$$
x(t)=x_{0}+I_{2}(t)+o(1) .
$$

Thus, in order to analyze the behavior of $x(t)$ as $\epsilon$ tends to 0 we need to identify the contribution of the term $I_{2}(t)$ to the limiting equation. In this section we use the bounds on the moments of $y(t)$ that we derived in section 3.3 to investigate precisely the limit of $I_{2}(t)$ defined by $(3.5 \mathrm{~b})$ as $\epsilon \rightarrow 0$.

We start with the regime $\gamma \in(0,2)$. We have the following.

Lemma 4.1. Let $x(t)$ be given by (3.1), and let $y(t)=\dot{x}(t)$ be given by (3.2). Suppose that conditions (2.9) and (2.12) with $\rho=1$ hold. Then for $\gamma \in(0,2)$ the term $I_{2}(t)$ given by $(3.5 \mathrm{~b})$ has the form

$$
I_{2}(t)=\int_{0}^{t} f(x(s)) A^{-1} d W(s)+H(t),
$$

where

$$
\mathbb{E}\left(\sup _{0 \leq t \leq T}\|H(t)\|^{2 p}\right) \leq C \epsilon^{(2-\gamma) p-\sigma}
$$

where $\sigma>0$ is arbitrarily small.

Proof. $I_{2}(t)$ is given by $(3.24)$ :

$$
I_{2}(t)=\int_{0}^{t} f(x(s)) A^{-1} d W(s)-\epsilon \widehat{J}_{1}(t)+\epsilon \widehat{J}_{2}(t) .
$$

We have that

$$
\mathbb{E}\left(\sup _{0 \leq t \leq T}\left\|\epsilon \widehat{J}_{1}(t)\right\|^{2 p}\right) \leq C \epsilon^{2 p-\sigma} .
$$

Furthermore, estimate (3.29) gives

$$
\mathbb{E}\left(\sup _{0 \leq t \leq T}\left\|\epsilon \widehat{J}_{2}(t)\right\|^{2 p}\right) \leq C \epsilon^{(2-\gamma) p-\sigma} .
$$

Estimate (4.1) follows from the above bounds. 
From our estimates on terms $I_{i}(t), i=1, \ldots, 4$, we anticipate that $x(t)$ converges in mean square, as $\epsilon \rightarrow 0$, to $X(t)$, which satisfies (2.16). The proof of this convergence is presented in section 5 .

Now we proceed with the case $\gamma \in[2, \infty)$. We have the following lemma.

LemMa 4.2. Let $x(t)$ be given by (3.1), and let $y(t)=\dot{x}(t)$ be given by (3.2). Suppose that conditions (2.7), (2.9), (2.10), and (2.13) hold. Then for $\gamma \in[2, \infty)$ the term $I_{2}(t)$ in (3.1) has the form

$$
\begin{aligned}
I_{2}(t)= & \int_{0}^{t} \nabla \cdot\left(f(x(s)) \Theta f^{T}(x(s))\right) d s-\int_{0}^{t} f(x(s)) \Theta \nabla \cdot f^{T}(x(s)) d s \\
& +\int_{0}^{t} f(x(s)) A^{-1} d W(s) \\
& -\epsilon^{\gamma-1} \int_{0}^{t} d f(x(s)) y(s) \eta(s) d s+H(t),
\end{aligned}
$$

where

$$
\mathbb{E}\left(\sup _{0 \leq t \leq T}\|H(t)\|^{2 p}\right) \leq C \epsilon^{2 p-\sigma}
$$

where $\sigma>0$ is arbitrarily small.

Proof. From (3.24) we have

$$
I_{2}(t)=\int_{0}^{t} \sum_{j=1}^{\infty} \frac{f_{i j}(x(s)) \sqrt{\lambda_{j}}}{\alpha_{j}} d \beta_{j}(s)-\epsilon \widehat{J}_{1}(t)+\epsilon \widehat{J}_{2}(t),
$$

with $\mathbb{E}\left(\sup _{0 \leq t \leq T}\left\|\epsilon \widehat{J}_{1}(t)\right\|^{2 p}\right) \leq C \epsilon^{2 p-\sigma} .{ }^{5}$ In order to study the term $\widehat{J}_{2}(t)$ we need another two integrations by parts. We apply the Itô formula to the function

$$
G_{i j k}(x, y, \eta)=f_{i j, k}(x) y_{k} \alpha_{j}^{-1} \eta_{j},
$$

from which we obtain, after some algebra,

$$
\begin{aligned}
\epsilon \frac{f_{i j, k} \eta_{j} y_{k}}{\alpha_{j}} d t= & -\epsilon^{\gamma+1} d\left(\frac{f_{i j, k} \eta_{j} y_{k}}{\alpha_{j}}\right)+\epsilon^{\gamma+1} \sum_{\ell=1}^{d} \frac{f_{i j, k \ell} \eta_{j} y_{k} y_{\ell}}{\alpha_{j}} d t \\
& +\epsilon \frac{f_{i j, k} \eta_{j} b_{k}}{\alpha_{j}} d t+\sum_{\rho=1}^{\infty} \frac{f_{i j, k} f_{k \rho} \eta_{j} \eta_{\rho}}{\alpha_{j}} d t \\
& -\epsilon^{\gamma} \frac{f_{i j, k} y_{k} \sqrt{\lambda_{j}}}{\alpha_{j}} d \beta_{j}-\epsilon^{\gamma-1} f_{i j, k} y_{k} \eta_{j} d t .
\end{aligned}
$$

Now we define the following functions (no summation):

$$
F_{i j k \rho}=f_{i j, k} f_{k \rho}
$$

and

$$
G_{i j k \rho}=F_{i j k \rho} \eta_{\rho} \eta_{j} \alpha_{j}^{-1}
$$

\footnotetext{
${ }^{5}$ This estimate is independent of $\gamma$.
} 
We apply the Itô formula to the function $G_{i j k \rho}$ to obtain

$$
\begin{aligned}
d G_{i j k \rho}= & \sum_{\ell} F_{i j k \rho, \ell} y_{\ell} \eta_{j} \eta_{\rho} \alpha_{j}^{-1} d t-\frac{1}{\epsilon^{2}} F_{i j k \rho} \alpha_{j}^{-1} \eta_{j} \eta_{\rho}\left(\alpha_{\rho}+\alpha_{j}\right) d t \\
& +\frac{1}{\epsilon^{2}} F_{i j k \rho} \alpha_{j}^{-1} \sqrt{\lambda_{j} \lambda_{\rho}} \delta_{j \rho} d t+\frac{1}{\epsilon} F_{i j k \rho} \alpha_{j}^{-1}\left(\eta_{\rho} \sqrt{\lambda_{j}} d \beta_{j}+\eta_{j} \sqrt{\lambda_{\rho}} d \beta_{\rho}\right),
\end{aligned}
$$

from which, after multiplying through by $\epsilon^{2}\left(\alpha_{j}+\alpha_{\rho}\right)^{-1}$ and taking the sum over $\rho=1,2, \ldots$, we get

$$
\begin{aligned}
\sum_{\rho=1}^{\infty} \frac{f_{i j, k} f_{k \rho} \eta_{j} \eta_{\rho}}{\alpha_{j}} d t= & \frac{f_{i j, k} f_{k j} \lambda_{j}}{2 \alpha_{j}^{2}} d t \\
& -\epsilon^{2} d\left(\sum_{\rho=1}^{\infty} \frac{f_{i j, k} f_{k \rho} \eta_{\rho} \eta_{j}}{\left(\alpha_{j}+\alpha_{\rho}\right) \alpha_{j}}\right)+\epsilon^{2} \sum_{\ell=1}^{d} \sum_{\rho=1}^{\infty} \frac{\left(f_{i j, k} f_{k \rho}\right)_{\ell} y_{\ell} \eta_{\rho} \eta_{j}}{\alpha_{j}\left(\alpha_{\rho}+\alpha_{j}\right)} d t \\
& +\epsilon \sum_{\rho=1}^{\infty} \frac{f_{i j, k} f_{k \rho}}{\alpha_{j}\left(\alpha_{\rho}+\alpha_{j}\right)}\left(\eta_{\rho} \sqrt{\lambda_{j}} d \beta_{j}+\eta_{j} \sqrt{\lambda_{\rho}} d \beta_{\rho}\right) .
\end{aligned}
$$

Note that

$$
f_{i j, k} f_{k j}=\left(f_{i j} f_{k j}\right)_{, k}-f_{i j} f_{k, k j} .
$$

Thus, from the above calculations, after taking the sum over $j \in \mathbb{Z}^{d}$ and $k=1, \ldots, d$, we obtain

$$
\begin{aligned}
\epsilon \widehat{J}_{2}(t)= & \int_{0}^{t} \nabla \cdot\left(f(x(s)) \Theta f^{T}(x(s))\right) d s-\int_{0}^{t} f(x(s)) \Theta \nabla \cdot f^{T}(x(s)) d s \\
& +\int_{0}^{t} f(x(s)) A^{-1} d W(s)-\epsilon^{\gamma-1} \int_{0}^{t} d f(x(s)) y(s) \eta(s) d s+H(t),
\end{aligned}
$$

where $H(t)=\sum_{\ell=1}^{8} H_{\ell}(t)$ with

$$
\begin{gathered}
H_{1}^{i}(t)=-\left.\epsilon^{2} \sum_{j, \rho=1}^{\infty} \sum_{k=1}^{d} \frac{f_{i j, k}(x(s)) f_{k \rho}(x(s)) \eta_{\rho}(s) \eta_{j}(s)}{\alpha_{j}\left(\alpha_{\rho}+\alpha_{j}\right)}\right|_{0} ^{t}, \\
H_{2}^{i}(t)=\epsilon^{2} \int_{0}^{t} \sum_{j, \rho=1}^{\infty} \sum_{k, \ell=1}^{d} \frac{\left(f_{i j, k \ell}(x(s)) f_{k \rho}(x(s))\right)_{, \ell} y_{\ell}(s) \eta_{\rho}(s) \eta_{j}(s)}{\alpha_{j}\left(\alpha_{j}+\alpha_{\rho}\right)} d s, \\
H_{3}^{i}(t)=\epsilon \int_{0}^{t} \sum_{j, \rho=1}^{\infty} \sum_{k=1}^{d} \frac{f_{i j, k}(x(s)) f_{k \rho}(x(s)) \eta_{\rho}(s) \sqrt{\lambda_{j}}}{\alpha_{j}\left(\alpha_{j}+\alpha_{\rho}\right)} d \beta_{j}(s), \\
H_{4}^{i}(t)=\epsilon \int_{0}^{t} \sum_{j, \rho=1}^{\infty} \sum_{k=1}^{d} \frac{f_{i j, k}(x(s)) f_{k \rho}(x(s)) \eta_{j}(s) \sqrt{\lambda_{\rho}}}{\alpha_{j}\left(\alpha_{j}+\alpha_{\rho}\right)} d \beta_{\rho}(s), \\
H_{5}^{i}(t)=-\left.\epsilon^{\gamma+1} \sum_{j=1}^{\infty} \sum_{k=1}^{d} \frac{f_{i j, k}(x(s)) y_{k}(s) \eta_{j}(s)}{\alpha_{j}}\right|_{0} ^{t}
\end{gathered}
$$




$$
\begin{gathered}
H_{6}^{i}(t)=\epsilon^{\gamma+1} \int_{0}^{t} \sum_{j=1}^{\infty} \sum_{k, \ell=1}^{d} \frac{f_{i j, k \ell}(x(s)) y_{k}(s) y_{\ell}(s) \eta_{j}(s)}{\alpha_{j}} d s, \\
H_{7}^{i}(t)=\epsilon \int_{0}^{t} \sum_{j=1}^{\infty} \sum_{k=1}^{d} \frac{f_{i j, k}(x(s)) \eta_{j}(s) b_{k}(x(s))}{\alpha_{j}} d s, \\
H_{8}^{i}(t)=\epsilon^{\gamma} \int_{0}^{t} \sum_{j=1}^{\infty} \sum_{k=1}^{d} \frac{f_{i j, k}(x(s)) y_{k}(s) \sqrt{\lambda_{j}}}{\alpha_{j}} d \beta_{j}(s) .
\end{gathered}
$$

Now we have to bound the terms $H_{\ell}(t), \ell=1, \ldots, 8$. The necessary estimates are proved in Lemma A.1 in the appendix. The final result is that

$$
\mathbb{E}\left(\sup _{0 \leq t \leq T}\|H(t)\|^{2 p}\right) \leq C \epsilon^{2 p-\sigma} .
$$

This completes the proof of the lemma.

Lemma 4.2, together with the estimates on $I_{3}(t)$ and $I_{4}(t)$, will enable us to show that for $\gamma \in(2, \infty)$ the particle position $x(t)$ converges in $L^{2 p}(\Omega, C([0, T] ; \mathbb{R}))$ to the solution of the Itô SDE (2.19). The precise convergence theorem will be proved in the next section.

The above argument fails when $\gamma=2$ since in this case we cannot control the last integral on the right-hand side of (4.2) uniformly in $\epsilon$. For the convergence theorem, in this case we need the following corollary of the previous lemma.

Corollary 4.3. Let the conditions of Lemma 4.2 be satisfied, and let $\gamma=2$. Then $I_{2}(t)$ has the following form:

$$
\begin{aligned}
I_{2}(t)= & \int_{0}^{t} \nabla \cdot\left(f(x(s)) \widehat{\Theta} f^{T}(x(s))\right) d s-\int_{0}^{t} f(x(s)) \widehat{\Theta} \nabla \cdot f^{T}(x(s)) d s \\
& +\int_{0}^{t} f(x(s)) A^{-1} d W(s)+\widehat{H}(t),
\end{aligned}
$$

with $\mathbb{E}\left(\sup _{0 \leq t \leq T}\|\widehat{H}(t)\|^{2 p}\right) \leq \epsilon^{2 p-\sigma}$, where $\sigma>0$ is arbitrarily small.

Proof. We set $\gamma=2$ in (4.4), solve for $f_{i j, k} y_{k} \eta_{j} \alpha_{j}^{-1} d t$ and combine the result with (4.5), sum over $j \in \mathbb{Z}^{d}$ and $k=1, \ldots, d$, and integrate over $[0, t]$ to obtain

$$
\begin{aligned}
I_{2}(t)= & \int_{0}^{t} \nabla \cdot\left(f(x(s)) \widehat{\Theta} f^{T}(x(s))\right) d s-\int_{0}^{t} f(x(s)) \widehat{\Theta} \nabla \cdot f^{T}(x(s)) d s \\
& +\int_{0}^{t} f(x(s)) A^{-1} d W(s)+\widehat{H}(t),
\end{aligned}
$$

with $\widehat{H}(t)=\sum_{k=1}^{8} \widehat{H}_{k}(t)$. The terms $\widehat{H}_{k}(t), k=1, \ldots, 8$, are similar to the terms defined in (4.6), with the difference that the diagonal operator $(I+A)^{-1}$ is applied to every one of them. Similar techniques to the ones used in the proof of the previous ones still apply. We obtain estimate $\mathbb{E}\left(\sup _{0 \leq t \leq T}\|\widehat{H}(t)\|^{2 p}\right) \leq C \epsilon^{2 p-\sigma}$, provided that conditions (2.13) hold.

The above corollary will enable us to show that, for $\gamma=2, x(t)$ converges to $X(t)$, which satisfies SDE (2.21). This leads to the surprising conclusion that in this case the correction to the drift is not the usual Stratonovich correction. The precise convergence theorem will be proved in the next section. 
5. Proofs of the convergence theorems. In this section we prove the convergence theorems, Theorems $2.3,2.4$, and 2.5. In the following proofs we will use the fact that $f: \ell_{2} \rightarrow \mathbb{R}^{d}$ is a Lipschitz continuous map, provided that condition (2.12b) with $\rho=\frac{1}{2}$ holds. In particular, a calculation similar to the one presented in (3.15) in the proof of Lemma 3.7 yields

$$
\left\|(f(X(s))-f(x(s))) A^{-1}\right\|_{L_{0}^{2}} \leq C\|X(s)-x(s)\| .
$$

Proof of Theorem 2.3. We combine (3.1), together with estimates (3.7) and (3.26), Corollary 3.13 , and Lemma 4.1 to write $x(t)$ in the form

$$
x(t)=x_{0}+\int_{0}^{t} f(x(s)) A^{-1} d W(s)+\int_{0}^{t} b(x(s)) d s+R_{1},
$$

with $\mathbb{E}\left(\sup _{0 \leq t \leq T}\left\|R_{1}\right\|^{2 p}\right) \leq C\left(\epsilon^{(2-\gamma) p-\sigma}+\epsilon^{\gamma p-\sigma}\right)$. Now we take the difference between $X(t)$ given by $(2.16)$ and $x(t)$ given by (5.2), raise it to the $2 p$ th power, take the expectation value of the supremum, and use the Burkholder-Davis-Gundy inequality and the Lipschitz continuity of $f(x), b(x)$, together with the estimate on $R_{1}$, to obtain

$$
\begin{aligned}
\mathbb{E}\left(\sup _{0 \leq t \leq T}\|X(t)-x(t)\|^{2 p}\right) \\
\leq C \int_{0}^{T} \mathbb{E}\left\|(f(X(s))-f(x(s))) A^{-1}\right\|_{L_{2}^{0}}^{2 p} d s \\
\quad+C \int_{0}^{T} \mathbb{E}\left(\sup _{0 \leq t \leq s}\|X(t)-x(t)\|^{2 p}\right) d s+C \mathbb{E}\left(\sup _{0 \leq t \leq T}\left\|R_{1}(t)\right\|^{2 p}\right) \\
\leq C\left(\epsilon^{(2-\gamma) p-\sigma}+\epsilon^{\gamma p-\sigma}\right)+C \int_{0}^{T} \mathbb{E}\left(\sup _{0 \leq t \leq s}\|X(t)-x(t)\|^{2 p}\right) d s .
\end{aligned}
$$

We apply now Gronwall's lemma to the above equation for the function

$$
\xi(T)=\mathbb{E}\left(\sup _{0 \leq t \leq T}\|X(t)-x(t)\|^{2 p}\right)
$$

to conclude the proof of Theorem 2.3.

Now we proceed with the convergence theorems for $\gamma \in[2, \infty)$. Let us consider the case $\gamma>2$.

Proof of Theorem 2.4. Consider the integral

$$
J(t)=\epsilon^{\gamma-1} \int_{0}^{t} d f(x(s)) y(s) \eta(s) d s .
$$

We use Lemma 3.10 with $\rho=0$ to deduce

$$
\mathbb{E}\left(\sup _{0 \leq t \leq T}\|J(t)\|^{2 p}\right) \leq C \epsilon^{2 p(\gamma-2)-\sigma}
$$

Now we combine (3.1) with estimates (3.7), (3.26), and (3.12a) and Lemma 4.2, together with the above estimate, to write $x(t)$ in the form

$$
x(t)=x_{0}+\int_{0}^{t} B(x(s)) d s+\int_{0}^{t} f(x(s)) A^{-1} d W(s)+R_{2}(t),
$$


with $\mathbb{E}\left(\sup _{0 \leq t \leq T}\left\|R_{2}(t)\right\|^{2}\right) \leq C\left(\epsilon^{2 p(\gamma-2)-\sigma}+\epsilon^{2 p-\sigma}\right)$ and

$$
B(x(s))=b(x(s))+\nabla \cdot\left(f(x(s)) \Theta f^{T}(x(s))\right)-f(x(s)) \Theta \nabla \cdot f^{T}(x(s)) .
$$

Now assumptions (2.9) and (2.7) imply that the drift term $B(x)$ in (5.3) is Lipschitz continuous:

$$
\|B(X)-B(x)\| \leq C\|X-x\|
$$

under condition (2.13c). The Lipschitz continuity of $B(x)$, together with (5.1) and the Burkholder-Davis-Gundy inequality, gives

$$
\begin{aligned}
\mathbb{E}\left(\sup _{0 \leq t \leq T}\|X(t)-x(t)\|^{2 p}\right) \leq & C_{1}\left(\epsilon^{2 p(\gamma-2)-\sigma}+\epsilon^{2 p-\sigma}\right) \\
& +C_{2} \int_{0}^{T} \mathbb{E}\left(\sup _{0 \leq t \leq s}\|X(t)-x(t)\|^{2 p}\right) d s
\end{aligned}
$$

from which Theorem 2.4 follows, upon applying Gronwall's lemma.

Now we are ready to present the convergence proof and theorem for the case $\gamma=2$. Since the proof is essentially the same as the one of Theorem 2.4, we will be brief.

Proof of Theorem 2.5. We combine (3.1) with estimates (3.7), (3.26), and (3.12a) and Corollary 4.3 to write $x(t)$ in the form, for $\gamma=2$,

$$
x(t)=x_{0}+\int_{0}^{t} \widehat{B}(x(s)) d s+\int_{0}^{t} f(x(s)) A^{-1} d W(s)+R_{3}(t),
$$

with $\mathbb{E}\left(\sup _{0 \leq t \leq T}\left\|R_{3}(t)\right\|^{2 p}\right) \leq C \epsilon^{2 p-\sigma}$ and

$$
\widehat{B}(x(s))=b(x(s))+\nabla \cdot\left(f(x(s)) \widehat{\Theta} f^{T}(x(s))\right)-f(x(s)) \widehat{\Theta} \nabla f^{T}(x(s)) .
$$

Assumptions (2.9), (2.7), and (2.13c) ensure that $\widehat{B}(x)$ is Lipschitz continuous. As in the proof of the previous theorem, we take the difference between $X(t)$ given by $(2.21)$ and $x(t)$ given by (5.5), raise it to the $2 p$ th power, take the expectation value of the supremum, and use the Burkholder-Davis-Gundy inequality and the Lipschitz continuity of the terms in (5.5) to obtain

$$
\mathbb{E}\left(\sup _{0 \leq t \leq T}\|X(t)-x(t)\|^{2 p}\right) \leq C_{1} \epsilon^{2 p-\sigma}+C_{2} \int_{0}^{T} \mathbb{E}\left(\sup _{0 \leq t \leq T}\|X(t)-x(t)\|^{2 p}\right) d s .
$$

Now we apply Gronwall's lemma to obtain estimate (2.22).

\section{Applications.}

6.1. Inertial particles in a random field. A model for the motion of inertial particles in turbulent flows was introduced in $[29,30]$. It consists of the Stokes law for the particle motion with the background divergence-free fluid velocity field being an infinite dimensional OU process. We assume that the motion takes place on the two-dimensional unit torus $\mathbb{T}^{2}$ :

$$
\begin{gathered}
\tau \ddot{x}=v(x, t)-\dot{x}, \\
v=\nabla^{\perp} \psi, \\
d \psi=\nu \Delta \psi d t+\sqrt{\nu} d W,
\end{gathered}
$$


where $\nabla^{\perp}:=\left(\frac{\partial}{\partial x_{2}},-\frac{\partial}{\partial x_{1}}\right)^{T}$ stands for the skew gradient and $\psi$ denotes the stream function. Furthermore, $W(x, t)$ denotes a $Q$-Wiener process on

$$
H:=\left\{f \in L_{p e r}^{2}\left(\mathbb{T}^{2}\right) ; \int_{\mathbb{T}^{2}} f d x=0\right\} .
$$

Various asymptotic limits for (6.1) were considered in [24]. Let us now consider the scaling limit considered in this paper.

We assume that $\tau=\tau_{0} \epsilon^{\gamma-1}$ and that the inverse noise correlation time $\nu$ is of $\mathcal{O}\left(\epsilon^{-1}\right)$, and we rescale time by $t \rightarrow t / \epsilon$. Moreover, we expand the solutions of (6.1c) in terms of the eigenfunctions of the Laplacian on $\mathbb{T}^{2}, e^{i k \cdot x}$. We also set $K=2 \pi \mathbb{Z}^{2} \backslash\{(0,0)\}$ and denote $\widehat{\mathbb{C}}^{K}:=\left\{\eta \in \mathbb{C}^{K} ; \eta_{k}=\bar{\eta}_{-k}\right\}$, equipped with the standard $\ell_{2}$ inner product. Setting $\tau_{0}=1$ for notational simplicity, the rescaled equations (6.1) can be written in the form

$$
\begin{gathered}
\epsilon^{\gamma} \ddot{x}=\frac{f(x) \eta(t)}{\epsilon}-\dot{x}(t), \\
f(x) \xi=\sum_{k \in K} i k^{\perp} e^{i k \cdot x} \xi_{k}, \\
d \eta_{k}=-\frac{1}{\epsilon^{2}}|k|^{2} \eta_{k} d t+\frac{1}{\epsilon} \sqrt{\lambda_{k}} d \beta_{k}, \quad k \in K,
\end{gathered}
$$

with $k^{\perp}=\left[k_{2}-k_{1}\right]^{T}, \eta=\left\{\eta_{k}\right\}_{k=1}^{\infty} \in \widehat{\mathbb{C}}^{K}$. Moreover, $\left\{\beta_{k}(t)\right\}_{k=1}^{\infty}$ are mutually independent one-dimensional standard Brownian motions satisfying the reality conditions $\beta_{k}=\bar{\beta}_{-k}$.

It was shown in [29] that

$$
f \Theta f^{T}=\sigma I \quad \text { with } \quad \sigma=\sum_{k \in K} \frac{\lambda_{k}}{2|k|^{2}},
$$

where $\Theta$ is defined in (2.18) and $I$ stands for the identity matrix. A similar calculation reveals that

$$
f \widehat{\Theta} f^{T}=\sigma I \quad \text { with } \quad \widehat{\sigma}=\sum_{k \in K} \frac{\lambda_{k}}{2|k|^{2}\left(1+|k|^{2}\right)}
$$

Furthermore, the incompressibility of the velocity field implies that

$$
\nabla \cdot f^{T}=0 .
$$

The above calculations imply that, for the inertial particles problem whose motion is modeled by (6.1), the Itô and Stratonovich interpretations of the stochastic integral coincide and that the limiting equation of motion is

$$
X(t)=x_{0}+\int_{0}^{t} f(X(s)) A^{-1} d W(s)
$$

for all values of $\gamma>0$. In fact, Theorems $2.3,2.4$, and 2.5 , together with the properties of the eigenfunctions of the Laplacian on $\mathbb{T}^{2}$, yield the following result. 
Proposition 6.1. Let $x(t)$ be the solution of $(6.2 \mathrm{a})$ and $\gamma \in(0, \infty)$. Assume that

$$
\sum_{k \in K} \sqrt{\lambda_{k}}<\infty \quad \text { for } \quad \gamma \in(0,2)
$$

and that

$$
\sum_{k \in K} \sqrt{\lambda_{k}}|k|<\infty \quad \text { for } \quad \gamma \in[2, \infty)
$$

Assume further that conditions (2.14) and (2.15) hold and that the initial conditions for $(6.2 \mathrm{c})$ are stationary. Then $x(t)$ converges, as $\epsilon \rightarrow 0$, to $X(t)$, which satisfies

$$
X(t)=x_{0}+\int_{0}^{t} f(X(s)) A^{-1} d W(s)
$$

with

$$
\begin{gathered}
\mathbb{E}\left(\sup _{0 \leq t \leq T}\|X(t)-x(t)\|^{2 p}\right) \leq C\left(\epsilon^{\gamma p}+\epsilon^{(2-\gamma) p-\sigma}\right) \quad \text { for } \quad \gamma \in(0,2), \\
\mathbb{E}\left(\sup _{0 \leq t \leq T}\|X(t)-x(t)\|^{2 p}\right) \leq C \epsilon^{2-\sigma} \quad \text { for } \quad \gamma=2,
\end{gathered}
$$

and

$$
\mathbb{E}\left(\sup _{0 \leq t \leq T}\|X(t)-x(t)\|^{2 p}\right) \leq C\left(\epsilon^{2 p-\sigma}+\epsilon^{2 p(\gamma-2)-\sigma}\right) \quad \text { for } \quad \gamma \in(2, \infty),
$$

where $\sigma>0$ is arbitrarily small. The constant $C$ depends on the moments of the initial conditions, the spectrum of the Wiener process, the operator $A$, the exponent $p$, the maximum time $T$, and $\sigma$.

Physically, we are looking at inertial particles in rapidly decorrelating velocity fields over long times. The parameter $\gamma$ affects the nondimensional mass of the particle, which is of $\mathcal{O}\left(\epsilon^{\gamma-1}\right)$. Provided that $\gamma>0$, which includes a range of massive, as well as light, particles, the limiting particle motion is equivalent in law to a Brownian motion; see (6.3). For $\gamma=0$, however, the limiting motion is that of the integrated OU process: the particle velocity is of OU type [24].

6.2. Diffusion in solids. Consider now the motion of a particle in one dimension under the influence of a periodic potential $V(x)$, subject to dissipation:

$$
\tau \ddot{x}=-V^{\prime}(x)-\dot{x} .
$$

We assume that the derivative of the potential can be written in the following Fourier sine series:

$$
V^{\prime}(x)=-\sum_{j=1}^{\infty} \sin (j x) \mu_{j} .
$$

We assume further that the control parameters $\mu_{j}$ are noisy and of the form

$$
\mu_{j}=\mu_{j}^{0}+\frac{1}{\epsilon} \eta_{j}\left(t / \epsilon^{2}\right)
$$


where $\left\{\mu_{j}^{0}\right\}_{j=1}^{\infty}$ are constants and $\left\{\eta_{j}(t)\right\}_{j=1}^{\infty}$ are one-dimensional OU processes driven by mutually independent noises:

$$
d \eta_{j}=-j^{2} \eta_{j} d t+\sqrt{\lambda_{j}} d \beta_{j}, \quad j=1, \ldots, \infty .
$$

Substituting the above into (6.4) and assuming that the particle relaxation time $\tau$ is of $\mathcal{O}\left(\epsilon^{\gamma}\right)$ we obtain

$$
\begin{gathered}
\epsilon^{\gamma} \ddot{x}=-V_{0}^{\prime}(x)-\dot{x}+\frac{1}{\epsilon} \sum_{j=1}^{\infty} \sin (j x) \eta_{j}(t), \\
d \eta_{j}=-\frac{1}{\epsilon^{2}} j^{2} \eta_{j} d t+\frac{1}{\epsilon} \sqrt{\lambda_{j}} d \beta_{j}, \quad j=1, \ldots, \infty,
\end{gathered}
$$

where $V_{0}^{\prime}(x)=-\sum_{j=1}^{\infty} \sin (j x) \mu_{j}^{0}$. We use now Theorems 2.3, 2.4, and 2.5 to deduce the following result.

Proposition 6.2. Let $x(t)$ be the solution of $(6.5)$ and $\gamma \in(0, \infty)$. Assume that

$$
\sum_{j=1}^{\infty} \sqrt{\lambda_{j}} j^{-1}<\infty \quad \text { for } \quad \gamma \in(0,2)
$$

and that

$$
\sum_{j=1}^{\infty} \sqrt{\lambda_{j}}<\infty \quad \text { for } \quad \gamma \in[2, \infty) .
$$

Assume further that conditions (2.14) and (2.15) hold, that $V_{0}(x) \in C_{b}^{2}(\mathbb{R})$, and that the initial conditions for $(6.2 \mathrm{c})$ are stationary. Then $x(t)$ converges, as $\epsilon \rightarrow 0$, to $X(t)$, which satisfies

$$
X(t)=\left\{\begin{aligned}
x_{0}- & V_{0}^{\prime}(x) \\
& +\int_{0}^{t} \sum_{j=1}^{\infty} \frac{\sqrt{\lambda_{j}}}{j^{2}} \sin (j X(s)) d \beta_{j}(s) \quad: \quad \gamma<2, \\
x_{0}- & V_{0}^{\prime}(x)+\frac{1}{4} \int_{0}^{t} \sum_{j=1}^{\infty} \frac{\lambda_{j}}{j^{3}\left(1+j^{2}\right)} \sin (2 j X(s)) d s \\
& +\int_{0}^{t} \sum_{j=1}^{\infty} \frac{\sqrt{\lambda_{j}}}{\alpha_{j}} \sin (j X(s)) d \beta_{j}(s) \quad: \quad \gamma=2, \\
x_{0}- & V_{0}^{\prime}(x)+\frac{1}{4} \int_{0}^{t} \sum_{j=1}^{\infty} \frac{\lambda_{j}}{j^{3}} \sin (2 j X(s)) d s \\
& +\int_{0}^{t} \sum_{j=1}^{\infty} \frac{\sqrt{\lambda_{j}}}{j^{2}} \sin (j X(s)) d \beta_{j}(s) \quad: \quad \gamma>2,
\end{aligned}\right.
$$

with

$$
\begin{gathered}
\mathbb{E}\left(\sup _{0 \leq t \leq T}\|X(t)-x(t)\|^{2 p}\right) \leq C\left(\epsilon^{\gamma p}+\epsilon^{(2-\gamma) p-\sigma}\right) \quad \text { for } \quad \gamma \in(0,2), \\
\mathbb{E}\left(\sup _{0 \leq t \leq T}\|X(t)-x(t)\|^{2 p}\right) \leq C \epsilon^{2 p-\sigma} \quad \text { for } \quad \gamma=2,
\end{gathered}
$$

and

$$
\mathbb{E}\left(\sup _{0 \leq t \leq T}\|X(t)-x(t)\|^{2 p}\right) \leq C\left(\epsilon^{2 p-\sigma}+\epsilon^{2 p(\gamma-2)-\sigma}\right) \quad \text { for } \quad \gamma \in[2, \infty),
$$


where $\sigma>0$ is arbitrarily small. The constant $C$ depends on the moments of the initial conditions, the spectrum of the Wiener process, the operator A, the exponent $p$, the maximum time $T$, and $\sigma$.

We remark that for $\gamma \in(0,2)$ the particle motion is in the mean potential

$$
V_{0}(x)=\mathbb{E}(V(x, t)) .
$$

On the other hand, for $\gamma \geq 2$, the limiting motion particle motion is in modified potential, which depends discontinuously on $\gamma$ as $\gamma \rightarrow 2^{+}$.

7. Conclusions. The Itô versus Stratonovich problem is studied in this paper for a class of infinite dimensional mean zero Gaussian random fields. It is shown that the correct interpretation of the stochastic integral in the limiting equation depends on the rate with which the particle relaxation time $\tau_{p}$ tends to 0 , relative to that of the noise correlation time $\tau_{n}$. In particular, it was shown that in the case where $\tau_{p}$ and $\tau_{n}$ tend to zero at the same rate, the limiting stochastic integral is neither of Itô nor of Stratonovich type.

The proof of our convergence theorems is based entirely on the pathwise techniques developed in [8] and used previously in [24], rather than the weak convergence methods of, e.g., [9]. Our techniques enable us to obtain strong, i.e., pathwise, convergence results, as well as sharp upper bounds on the convergence rates. A drawback of the method employed in this paper is that it is applicable only for noise processes which can be expressed as solutions of SDEs, like the one used in this paper. In order to apply the results reported in this paper to more general classes of colored approximations to white noise, weak convergence techniques will be more appropriate.

Appendix A. Estimates on terms $\boldsymbol{H}_{i}(t), i=1, \ldots, 8$. In this appendix we prove the following lemma.

Lemma A.1. Consider the terms $H_{i}(t), i=1, \ldots, 8$, defined in (4.6), and set $H(t)=\sum_{i=1}^{8} H_{i}(t)$. Assume that conditions (2.7), (2.13a), (2.9), (2.10), and (2.12a) with $\rho=\frac{1}{2}$ and $(2.12 \mathrm{~b})$ with $\rho=0$ hold. Then the following estimate holds:

$$
\mathbb{E}\left(\sup _{0 \leq t \leq T}\|H(t)\|^{2 p}\right) \leq C \epsilon^{2 p} .
$$

Proof. We start with $H_{1}(t)$. First, we compute

$$
\begin{aligned}
& \left|\sum_{j, \rho=1}^{\infty} \sum_{k=1}^{d} \frac{f_{i j, k}(x(s)) f_{k \rho}(x(s)) \eta_{\rho}(s) \eta_{j}(s)}{\alpha_{j}\left(\alpha_{\rho}+\alpha_{j}\right)}\right| \\
& \leq C \sum_{\rho=1}^{\infty} \frac{\alpha_{\rho}^{\alpha+r}\left|\eta_{\rho}(s)\right|}{\alpha_{\rho}} \sum_{j=1}^{\infty} \frac{\alpha_{j}^{\beta+r}\left|\eta_{j}(s)\right|}{\alpha_{j}} \\
& \leq C\left(\sum_{\rho=1}^{\infty} \alpha_{\rho}^{\alpha+r-1}\left|\eta_{\rho}(s)\right|\right)^{2}+C\left(\sum_{j=1}^{\infty} \alpha_{j}^{\beta+r-1}\left|\eta_{j}(s)\right|\right)^{2} \\
& =: J_{1}(t)+J_{2}(t) .
\end{aligned}
$$

Now calculations similar to the ones employed in the proof of Lemma 3.2 enable us to obtain

$$
\mathbb{E}\left(\sup _{0 \leq t \leq T}\left\|J_{1}(t)\right\|^{2 p}\right) \leq C \epsilon^{-\sigma}, \quad \mathbb{E}\left(\sup _{0 \leq t \leq T}\left\|J_{2}(t)\right\|^{2 p}\right) \leq C \epsilon^{-\sigma},
$$


for $\sigma>0$ is arbitrarily small, provided that conditions (2.12b) with $\rho=1$ and (2.12a) with $\rho=1$ hold. We use the above estimate and the definition of $H_{1}(t)$ to conclude:

$$
\mathbb{E}\left(\sup _{0 \leq t \leq T}\left\|H_{1}(t)\right\|^{2 p}\right) \leq C \epsilon^{4 p-\sigma}
$$

for $\sigma>0$ is arbitrarily small.

We proceed now with $H_{2}(t)$. We define

$$
J^{i}(t):=\sum_{j, \rho=1}^{\infty} \sum_{k, \ell=1}^{d} \frac{\left(f_{i j, k \ell}(x(s)) f_{k \rho}(x(s))\right)_{, \ell} y_{\ell}(s) \eta_{\rho}(s) \eta_{j}(s)}{\alpha_{j}\left(\alpha_{j}+\alpha_{\rho}\right)} .
$$

Now we compute

$$
\begin{aligned}
J^{i}(t)= & \sum_{j, \rho=1}^{\infty} \sum_{k, \ell=1}^{d} \frac{f_{i j, k \ell \ell}(x(s)) f_{k \rho}(x(s)) y_{\ell}(s) \eta_{\rho}(s) \eta_{j}(s)}{\alpha_{j}\left(\alpha_{j}+\alpha_{\rho}\right)} \\
& +\sum_{j, \rho=1}^{\infty} \sum_{k, \ell=1}^{d} \frac{f_{i j, k \ell}(x(s)) f_{k \rho, \ell}(x(s)) y_{\ell}(s) \eta_{\rho}(s) \eta_{j}(s)}{\alpha_{j}\left(\alpha_{j}+\alpha_{\rho}\right)} \\
\leq & C \sum_{j=1}^{\infty} \frac{\alpha_{j}^{\delta+r}\|y(s)\|\left|\eta_{j}(s)\right|}{\alpha_{j}} \sum_{\rho=1}^{\infty} \frac{\alpha_{\rho}^{\alpha+r}\left|\eta_{\rho}(s)\right|}{\alpha_{\rho}} \\
& +C \sum_{j=1}^{\infty} \frac{\alpha_{j}^{\gamma+r}\|y(s)\|\left|\eta_{j}(s)\right|}{\alpha_{j}} \sum_{\rho=1}^{\infty} \frac{\alpha_{\rho}^{\beta+r}\left|\eta_{\rho}(s)\right|}{\alpha_{\rho}} \\
\leq & C \epsilon^{\zeta}\|y(s)\|^{2}\left[\left(\sum_{j=1}^{\infty} \alpha_{j}^{\delta+r-1}\left|\eta_{j}(s)\right|\right)^{2}+\left(\sum_{j=1}^{\infty} \alpha_{j}^{\gamma+r-1}\left|\eta_{j}(s)\right|\right)^{2}\right] \\
& +C \epsilon^{-\zeta}\left[\left(\sum_{\rho=1}^{\infty} \alpha_{\rho}^{\alpha+r-1}\left|\eta_{\rho}(s)\right|\right)^{2}+\left(\sum_{\rho=1}^{\infty} \alpha_{\rho}^{\beta+r-1}\left|\eta_{\rho}(s)\right|\right)^{2}\right]
\end{aligned}
$$

for $\zeta \in \mathbb{R}$. We use now calculations similar to those used in order to prove Lemma 3.2 and estimate (3.13), together with Lemma 3.9, to deduce

$$
\mathbb{E}\left(\sup _{0 \leq t \leq T}\left\|H_{2}(t)\right\|^{2 p}\right) \leq C\left(\epsilon^{2 p \zeta-\sigma}+\epsilon^{4 p-2 p \zeta-\sigma}\right),
$$

provided that conditions (2.12) with $\rho=1$ hold. We now choose $\zeta=1$ to obtain

$$
\mathbb{E}\left(\sup _{0 \leq t \leq T}\left\|H_{2}\right\|^{2 p}\right) \leq C \epsilon^{2 p-\sigma} .
$$

Consider now the term $H_{4}(t)$. We introduce the cylindrical Wiener process

$$
\widehat{W}(t)=\sum_{\rho=1}^{\infty} \widehat{e}_{k} \beta_{k}(t) \text {. }
$$

Now we can write $H_{4}(t)$ in the form

$$
H_{4}(t)=\epsilon \int_{0}^{t} \widehat{F} d \widehat{W}
$$


where the map $\widehat{F}: \ell_{2} \rightarrow \mathbb{R}^{d}$ is defined as

$$
\{\widehat{F} \gamma\}_{i}=\sum_{j, \rho=1}^{\infty} \sum_{k=1}^{d} \frac{f_{i j, k}(x(s)) f_{k \rho}(x(s)) \eta_{j}(s) \sqrt{\lambda_{\rho}}}{\alpha_{j}\left(\alpha_{j}+\alpha_{\rho}\right)} \gamma_{\rho}, \quad i=1, \ldots, d \quad \forall \gamma \in \ell_{2}
$$

We need to estimate the Hilbert-Schmidt norm of $\widehat{F}$. We have

$$
\begin{aligned}
\|\widehat{F}\|_{L_{2}\left(\ell_{2}, \mathbb{R}^{d}\right)}^{2} & =\sum_{\rho=1}^{\infty} \sum_{i=1}^{d}\left|\sum_{k=1}^{d} \sum_{j=1}^{\infty} \frac{f_{i j, k}(x(s)) f_{k \rho}(x(s)) \eta_{j}(s) \sqrt{\lambda_{\rho}}}{\alpha_{j}\left(\alpha_{j}+\alpha_{\rho}\right)}\right|^{2} \\
& \leq C \sum_{\rho=1}^{\infty}\left(\sum_{j=1}^{\infty} \frac{\alpha_{j}^{\beta+r} \alpha_{\rho}^{\alpha+r}\left|\eta_{j}\right| \sqrt{\lambda_{\rho}}}{\alpha_{j}\left(\alpha_{j}+\alpha_{\rho}\right)}\right)^{2} \\
& \leq C \sum_{\rho=1}^{\infty}\left(\alpha_{\rho}^{\alpha+r-1} \sqrt{\lambda_{\rho}}\right)^{2}\left(\sum_{j=1}^{\infty} \alpha_{j}^{\beta+r-1}\left|\eta_{j}\right|\right)^{2} \\
& \leq C\left(\sum_{j=1}^{\infty} \alpha_{j}^{\beta+r-1}\left|\eta_{j}\right|\right)^{2}
\end{aligned}
$$

provided that condition (2.12a) with $\rho=\frac{1}{2}$ holds. We use now the Burkholder-DavisGundy and Hölder inequalities, together with a calculation similar to the one used in the proof of Lemma 3.2, to deduce

$$
\mathbb{E}\left(\sup _{0 \leq t \leq T}\left\|H_{4}(t)\right\|^{2 p}\right) \leq C \epsilon^{2 p} \int_{0}^{T} \mathbb{E}\|\widehat{F}\|_{L_{2}\left(\ell_{2}, \mathbb{R}^{d}\right)}^{2 p} d s \leq C \epsilon^{2 p},
$$

provided that condition (2.12b) with $\rho=1$ holds. Exactly the same analysis provides us with the estimate

$$
\mathbb{E}\left(\sup _{0 \leq t \leq T}\left\|H_{3}(t)\right\|^{2 p}\right) \leq C \epsilon^{2 p}
$$

under conditions (2.12b) with $\rho=\frac{1}{2}$ and (2.12a) with $\rho=1$.

Now we consider term $H_{5}(t)$. We have

$$
H_{5}(t)=-\left.\epsilon^{\gamma+1} d f(x(s)) y(s) A^{-1} \eta(s)\right|_{0} ^{t}
$$

Now the calculations used in the proof of Lemma 3.10, together with (3.13), yield

$$
\begin{aligned}
\mathbb{E}\left(\sup _{0 \leq t \leq T}\left\|H_{5}(t)\right\|^{2 p}\right) & \leq C \epsilon^{2 p(\gamma+1)} \mathbb{E}\left(\sup _{0 \leq t \leq T}\left\|d f(x(s)) y(s) A^{-1} \eta(s)\right\|^{2 p}\right) \\
& \leq C \epsilon^{2 \gamma p-\sigma}
\end{aligned}
$$

where $\sigma>0$ is arbitrarily small, provided that (2.12b) holds with $\rho=1$.

Now we proceed with $H_{6}(t)$. We use a simple variant of (3.13) and assume (2.13a) 
WHITE NOISE LIMITS

to compute

$$
\begin{aligned}
\mathbb{E}\left(\sup _{0 \leq t \leq T}\left\|H_{6}(t)\right\|^{2 p}\right) & \leq C \epsilon^{2 p(\gamma+1)} \int_{0}^{T} \mathbb{E} \sum_{i=1}^{d}\left|\sum_{j=1}^{\infty} \sum_{k, \ell=1}^{d} \frac{f_{i j, k \ell}(x(s)) y_{k}(s) y_{\ell}(s) \eta_{j}(s)}{\alpha_{j}}\right|^{2 p} d s \\
& \leq C \epsilon^{2 p(\gamma+1)} \int_{0}^{T} \mathbb{E}\left(\|y(s)\|^{4 p}\left(\sum_{j=1}^{\infty} \alpha_{j}^{\alpha+\gamma-1} \eta_{j}(s)\right)^{2 p}\right) d s \\
& \leq C \epsilon^{2 p(\gamma-1)-\sigma}
\end{aligned}
$$

where $\sigma>0$.

Consider now $H_{7}(t)$. This term can be written in the following form:

$$
H_{7}(t)=\epsilon \int_{0}^{t} d f(x(s)) b(s) A^{-1} \eta(s) d s .
$$

We use the Hölder inequality, together with a calculation similar to the one presented in the proof of Lemma 3.10, as well as assumptions (2.9) and (2.12a) with $\rho=1$, to obtain

$$
\begin{aligned}
\mathbb{E}\left(\sup _{0 \leq t \leq T}\left\|H_{7}(t)\right\|^{2 p}\right) & \leq C \epsilon^{2 p} \int_{0}^{T} \mathbb{E}\left\|d f(x(s)) b(x(s)) A^{-1} \eta(s)\right\|^{2 p} d s \\
& \leq C \epsilon^{2 p}\|b(x)\|_{L^{\infty}\left(\mathbb{R}^{d}\right)}^{2 p} \int_{0}^{T} \mathbb{E}\left(\sum_{j=1}^{\infty} \alpha^{\beta+r-1}\left|\eta_{j}(s)\right|\right)^{2 p} d s \\
& \leq C \epsilon^{2 p}
\end{aligned}
$$

Finally, consider $H_{8}(t)$. We write it in the form

$$
H_{8}(t)=\epsilon^{\gamma} \int_{0}^{t} d f(x(s)) y(s) A^{-1} d W(s)
$$

We use the Burkholder-Davis-Gundy inequality to obtain

$$
\mathbb{E}\left(\sup _{0 \leq t \leq T}\left\|H_{8}(t)\right\|^{2 p}\right) \leq C \epsilon^{2 \gamma p} \mathbb{E}\left(\int_{0}^{T}\left\|d f(x(s)) y(s) A^{-1}\right\|_{L_{2}^{0}}^{2}\right)^{p} .
$$

Now we have

$$
\left\|d f(x(s)) y(s) A^{-1}\right\|_{L_{2}^{0}}^{2} \leq C\|y(s)\|^{2},
$$

provided that condition $(2.12 \mathrm{~b})$ with $\rho=0$ holds. Thus

$$
\mathbb{E}\left(\sup _{0 \leq t \leq T}\left\|H_{8}(t)\right\|^{2 p}\right) \leq C \epsilon^{2 \gamma p} \mathbb{E}\|y(t)\|^{2 p} \leq C \epsilon^{2 p(\gamma-1)-\sigma} .
$$

Now putting all the above estimates together we obtain (A.1). 
Acknowledgments. The authors are grateful to D. Cai, P. R. Kramer, and J. C. Mattingly for useful suggestions. They are particularly grateful to J. M. Sancho for useful suggestions and for providing them with $[27,28]$.

\section{REFERENCES}

[1] R. J. Adler, An Introduction to Continuity, Extrema, and Related Topics for General Gaussian Processes, IMS Lecture Notes Monogr. Ser. 12, Institute of Mathematical Statistics, Hayward, CA, 1990.

[2] L. Arnold, Stochastic Differential Equations: Theory and Applications, Wiley-Interscience, New York, 1974.

[3] R. Aurich, A. Bäcker, R. Schubert, and M. Taglieber, Maximum norms of chaotic quantum eigenstates and random waves, Phys. D, 129 (1999), pp. 1-14.

[4] G. Blankenship and G. C. Papanicolaou, Stability and control of stochastic systems with wide-band noise disturbances. I, SIAM J. Appl. Math., 34 (1978), pp. 437-476.

[5] R. Bouc And É. PARdoux, Asymptotic analysis of PDEs with wide-band noise disturbances, and expansion of the moments, Stochastic Anal. Appl., 2 (1984), pp. 369-422.

[6] R. A. Carmona AND J-P. Fouque, Diffusion-approximation for the advection-diffusion of a passive scalar by a space-time Gaussian velocity field, in Seminar on Stochastic Analysis, Random Fields and Applications (Ascona, 1993), Progr. Probab. 36, Birkhäuser, Basel, 1995, pp. 37-49.

[7] R. A. Carmona and L. Xu, Homogenization theory for time-dependent two-dimensional incompressible Gaussian flows, Ann. Appl. Probab., 7 (1997), pp. 265-279.

[8] R. M. Dowell, Differentiable Approximation to Brownian Motion on Manifolds, Ph.D. thesis, University of Warwick, Coventry, UK, 1980.

[9] S. N. Ethier and T. G. Kurtz, Markov Processes, Wiley Ser. Probab. Math. Statist. Probab. Math. Statist., John Wiley and Sons, New York, 1986.

[10] A. C. Fannjiang, Convergence of passive scalar fields in Ornstein-Uhlenbeck flows in Kraichnan's model, J. Statist. Phys., 114 (2004), pp. 115-135.

[11] J. García-Ojalvo And J. M. Sancho, Noise in Spatially Extended Systems, Inst. Nonlinear Sci., Springer-Verlag, New York, 1999.

[12] D. Givon And R. Kupferman, White noise limits for discrete dynamical systems driven by fast deterministic dynamics, Phys. A, 335 (2004), pp. 385-412.

[13] R. Graham and A. Schenzle, Stabilization by multiplicative noise, Phys. Rev. A (3), 26 (1982), pp. 1676-1685.

[14] D. GRIESER, Uniform bounds for eigenfunctions of the Laplacian on manifolds with boundary, Comm. Partial Differential Equations, 27 (2002), pp. 1283-1299.

[15] W. Horsthemke And R. Lefever, Noise-Induced Transitions. Theory and Applications in Physics, Chemistry, and Biology, Springer Ser. Synergetics 15, Springer-Verlag, Berlin, 1984.

[16] R. H. Kraichnan, Diffusion by a random velocity field, Phys. Fluids, 13 (1970), pp. 22-31.

[17] P. R. KRAMER, Two different rapid decorrelation in time limits for turbulent diffusion, J. Statist. Phys., 110 (2003), pp. 83-136.

[18] R. Kupferman, G. A. Pavliotis, And A. M. Stuart, Itô versus Stratonovich white noise limits for systems with inertia and colored multiplicative noise, Phys. Rev. E (3), 70 (2004), 036120 .

[19] H. J. Kushner And H. Huang, Approximating multiple Itô integrals with "band limited" processes, Stochastics, 14 (1985), pp. 85-113.

[20] H. J. Kushner And H. HuAng, Limits for parabolic partial differential equations with wide band stochastic coefficients and an application to filtering theory, Stochastics, 14 (1985), pp. 115-148.

[21] S. E. Mangioni, R. R. Deza, R. Toral, and H. S. Wio, Nonequilibrium phase transitions induced by multiplicative noise: Effects of self-correlation, Phys. Rev. E (3), 61 (2000), pp. $223-232$.

[22] E. Nelson, Dynamical Theories of Brownian Motion, Princeton University Press, Princeton, NJ, 1967.

[23] B. OKsendal, Stochastic Differential Equations, Springer-Verlag, Berlin, Heidelberg, New York, 1998.

[24] G. A. Pavliotis And A. M. Stuart, White noise limits for inertial particles in a random field, Multiscale Model. Simul., 1 (2003), pp. 527-553.

[25] G. Da Prato And J. ZABCZYK, Stochastic Equations in Infinite Dimensions, Encyclopedia Math. Appl. 44, Cambridge University Press, Cambridge, UK, 1992. 
[26] P. Reimann, Brownian motors: Noisy transport far from equilibrium, Phys. Rep., 361 (2002), pp. $57-265$.

[27] J. M. Sancho, M. San Miguel, And D. Dürr, Adiabatic elimination for systems of Brownian particles with nonconstant damping coefficients, J. Statist. Phys., 28 (1982), pp. 291-305.

[28] J. M. SANChO AND A. SANChez, External fluctuations in front dynamics with inertia: The overdamped limit, Eur. Phys. J. B Condens. Matter Phys., 16 (2000), pp. 127-131.

[29] H. Sigurgeirsson And A. M. Stuart, Inertial particles in a random field, Stoch. Dyn., 2 (2002), pp. 295-310.

[30] H. Sigurgeirsson And A. M. Stuart, A model for preferential concentration, Phys. Fluids, 14 (2002), pp. 4352-4361.

[31] C. D. Sogge, Fourier Integrals in Classical Analysis, Cambridge Tracts in Math. 105, Cambridge University Press, Cambridge, UK, 1993.

[32] E. Wong And M. ZAKaI, On the convergence of ordinary integrals to stochastic integrals, Ann. Math. Statist., 36 (1965), pp. 1560-1564.

[33] J. Zabczyk and G. Tessitore, Wong-Zakai Approximations of Stochastic Evolution Equations, Warwick Preprint 9, University of Warwick, Coventry, UK, 2001. 